\title{
Microneedle, bio-microneedle and bio-inspired microneedle: a review
}

\author{
Guojun Ma, Chengwei Wu
}

State Key Laboratory of Structural Analysis for Industrial Equipment, Department of

Engineering Mechanics, International Center for Computational Mechanics, Faculty

of Vehicle Engineering and Mechanics, Dalian University of Technology, Dalian,

116024, People's Republic of China

Corresponding author: cwwu@dlut.edu.cn 
Abstract: Microneedles (MNs) are micro-scale needles used for drug delivery and other targets. Micro-scale size endows them with many advantages over hypodermic needles, including painlessness, minimal invasiveness and convenient operation, but it may also lead to risk of mechanical failures, which should be prevented in the clinical applications of MNs. The objective of this review is mainly to introduce studies on the mechanics problems with respect to MNs. Firstly, the basic knowledge of MNs is introduced in brief, so that readers can understand the basic characteristics of MNs. Secondly, researches on inserting behavior and mechanical performances of MNs are discussed. Thirdly, literatures on the drug delivery and the pain resulted from the insertion of MNs are overviewed. Finally, some bio-microneedles and bio-inspired MNs are introduced.

Keywords: microneedle, bio-microneedle, transdermal drug delivery, insertion force.

\section{Introduction}

Oral administration of drugs is one of the most convenient methods of drug delivery, but sometimes it is not feasible because a few drugs may be poorly absorbed or degraded due to the enzymatic degradation in the gastrointestinal tract or liver [1]. As a common alternative to oral delivery of drugs, injection using a hypodermic needle has appeared over one hundred years. However, hypodermic needle injection will induce pain and invasiveness, produce sharp and bio-hazardous wastes, and require trained administer. Moreover, in the case of vaccine delivery, hypodermic needles are penetrated into muscle, where the immunologically reaction is weaker than that in the skin [2,3]. Transdermal drug delivery (TDD) is an alternative to the two methods mentioned above. Compared with oral and hypodermic administration, TDD can avoid hepatic first pass metabolism, as well as, it can be self-administrated in a non-invasive and painless manner [4]. However, the stratum corneum (SC), outmost 10-15 $\mu$ m layer of skin formed by dead corneocytes, forms a primary barrier for TDD [5]. This will seriously decrease the drug delivery efficiency and limit the types of drugs transported by TDD. Until 2011, only about 20 drugs have been approved by the Food and Drug Administration (FDA) in the USA as transdermal patches. Moreover, all these drug molecular weights are below 400 Da [6]. In 1976, a new technology named microneedle(s) [7] was firstly introduced to overcome the limitations of conventional TDD. After 1990s, with the development of contemporary microfabrication techniques, a great variety of MNs have been successfully fabricated. The MNs have typical lengths ranging from $25 \mu \mathrm{m}$ to 
$2000 \mu \mathrm{m}[8]$ and have much sharper tips than those of the hypodermic needles, so they can be used to disrupt the SC and form micro-scale drug delivery channels without touching the nerve fibres and blood vessels that are located in the epidermis and the dermis of the skin layer [9]. As a result, the drug delivery efficiency can be dramatically enhanced, as well as the types of drug transported by TDD can be significantly increased in a painless and minimally invasive manner [8-11].

Now, the MN technologies have increasingly grown for four decades. Numerous preclinical studies and limited number of clinical tests show that MNs can be used to deliver oligonucleotides, desmopressin, DNA, vaccines, insulin and human growth hormone through the skin [12]. Additionally, MNs was also widely studied for blood sampling [12-14], signal monitoring [15], intrascleral drug delivering [16], microneedle-assisted gene transferring [17], and biosensor [18-20]. This means that the MNs will target not only to the $\$ 32$ billion transdermal drug delivery and $\$ 25$ billion global vaccine markets but also to the over $\$ 120$ billion global biologics market [12]. Especially, MNs are considered to be the most powerful technology for vaccine delivery in developing countries [21]. The details of the functions and applications of MNs can be found in some comprehensive reviews [4,11,22-24], and the fabrication technologies of MNs can also been found in some review papers $[4,8,11,25]$. In this review, however, we will discuss some fundamental problems that may influence the integrated properties of MNs.

It is well known that MNs should have appropriate combination of mechanical strength, toughness and hardness to disrupt the SC without fracture and buckling failure. On the other hand, MN size must be small enough to ensure the painlessness and minimal invasiveness. Moreover, the drug delivery efficiency should also be fully taken into account at MN design. The factors accountable for MN mechanical properties are complicated, including materials, geometry and size, and needle density. In general, these factors will also influence the drug transportation efficiency, the extent of pain and invasiveness induced by MNs. In order to design 'intelligent' or 'optimized' MNs with painlessness, minimal invasiveness and high reliability, the intricate interplay of these factors should be fully understood, i.e., the mechanics problems concerning MN design should be deliberately studied. Although there have already been many works on this topic, to the best of our knowledge, there is no a comprehensive review on these literatures. Therefore, in the present review we pay our attentions on the literatures of this topic. Firstly, the types of MNs are briefly introduced. Secondly, studies on the mechanics problems of MN are discussed. Finally, researches on bio-microneedles and bio-inspired MNs are reviewed. 


\section{Classification of MNs}

\subsection{Categorized by material}

In general, MNs can be made of a wide variety of materials. Until now scientists have developed silicon MNs [9,26,27], glass MNs [28-31], ceramic MNs [32,33] metal MNs [34-37], hydrogel MNs [38,39], polymer MNs [40-47] and sugar MNs [48-50], etc. Because the micro-fabrication techniques for silicon have been developed for several decades, most of the MNs were made of silicon in the early developing stage. Furthermore, since silicon has relative high hardness, the silicon MNs are stiff enough to easily pierce into the skin. Henry et al. [9] fabricated $150 \mu \mathrm{m}$ long silicon MNs using a reactive ion etching technique. Experimental results showed that the permeability of human skin in vitro could be dramatically increased by up to 4 orders of magnitude after pierced by MNs. This work is usually considered as the first report on the use of MNs to enhance drug delivery across skin. In spite of these advantages, however, the manufacturing methods of silicon are relatively expensive and require clean room processing [51]. Moreover, because silicon is fragile material, silicon MNs are prone to fracture in transportation and application. Experiments carried out by Henry et al. [9] showed that a few MNs may break near the tip (top 5-10 $\mu \mathrm{m}$ ) after inserting into skin. These minimal silicon-fragments remaining underneath the skin may cause bad immunogenic consequences due to the unproven bio-compatibility of silicon [52]. Generally, MNs made out of brittle materials like silicon, such as ceramic, glass and so on, may all meet the similar problem. Therefore, whether these types of MNs can be used in clinic is still an open question to discuss. In contrast, some metals, such as stainless-steel, titanium, nickel, palladium, etc., usually have quite well integrated mechanical properties, including high toughness, strength, and hardness, which can protect MNs against mechanical failure. Additionally, compared with silicon MNs, metal MNs can be manufactured at relatively low cost using a variety of methods, such as electroplating, photochemical etching, micromilling and laser cutting, etc. Although some metals such as stainless-steel and titanium have been safely used in medical treatment for decades, the safety of other metals should be further investigated. Moreover, metal MNs will produce bio-hazardous tip wastes.

In comparison to silicon-like brittle materials, polymers usually have high toughness to enable the polymer MNs to avoid brittle fracture during their insertion into the skin or other tissues [44]. Because most polymers are bio-compatible, polymer MNs hardly induce harsh side effects. Moreover, some polymers are biodegradable or water-soluble, so drugs can be encapsulated in these dissolvable MNs. After insertion into the skin, drugs will release with the degradation or dissolution of these dissolvable MNs. This means that there are no sharp bio-hazardous tip wastes to be disposed. It is noteworthy that due to the relatively low melt temperature of most polymers 
some processing techniques, such as micro-molding technique, are fit for mass production of MNs at low cost. Hence, polymers are receiving increasing attentions and are considered as the most promising materials for MN fabrication [44]. However, there are also some problems with respect to polymer MNs. One of the knotty problems is that most polymers are soft. This probably induces catastrophic buckling failure of MNs during their insertion process. Especially in the case of blood sampling [13,14,53], where the MNs are much longer than those for drug delivery, buckling is easier to take place. Recently, Chu \& Prausnitz [54] fabricated a type of MN array with sharp-tipped dissolvable polymer arrowheads supported by blunt metal shafts as shown in Fig. 1 . Obviously, this is an intelligent design making the best of the advantages of metal and polymer, respectively. However, this design needs a complicated processing and additional cost, so further work concerning polymer MNs should be developed.
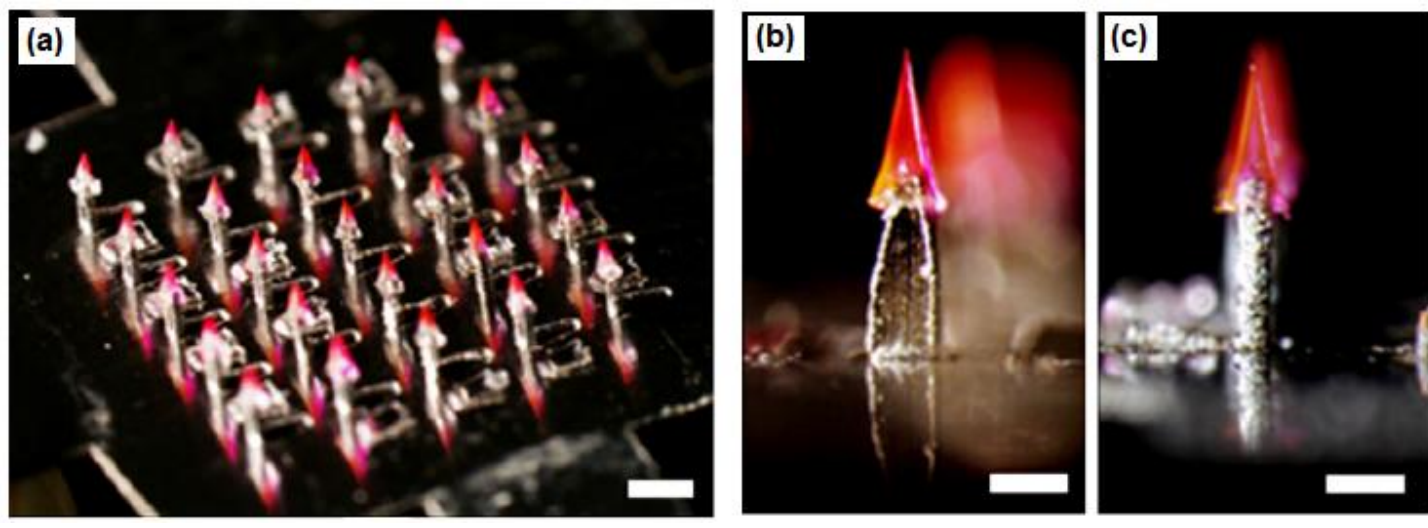

Fig. 1 Separable arrowhead MNs. (a) $5 \times 5$ array of separable arrowhead microneedles comprising $600 \mu$ m-tall metal shafts capped with $600 \mu \mathrm{m}$-tall watersoluble PVA/PVP arrowheads, Scale bar=1mm. (b) Individual needles are shown from the front. Scale bar=1 $\mathrm{mm}$ (c) side view, Scale bar=300 $\mu \mathrm{m}$. Using this type of MNs to delivery drug, there will be no sharp waste tips. Images are adapted from Ref. [54].

\subsection{Categorized by structure}

Based on the outside structure, MNs can be categorized as in-plane MNs [55] and out-of-plane MNs [9]. The in-plane MNs indicate that the length of MN is parallel to substrate plane (see Fig.2a), and the longitudinal axis of out-of-plane MN is perpendicular to the substrate plane (see Fig. 2b). From the viewpoint of processing, the density of out-of-plane MN array can reach a much high quantity [56], which is probably helpful to increase the drug-delivery efficiency, but it is difficult to fabricate too long out-of-plane MNs using such as etching or molding methods. In contrast, the density of in-plane MN array cannot be much high, but the length of them can be easily controlled [55]. Of course, the in-plane MNs can be converted into out-of-plane MNs using 
some special approaches [57-59]. For example, Zuck [57] fabricated in-plane MNs based on the combination of photolithography and chemical etching process, and then obtained out-of-plane MNs by microprotrusion method, i.e., through mechanically deforming the in-plane MNs. Cormier et al. [58] employed the similar method to convert the in-plane MNs into out-of-plane MNs. Oh et al. [59] firstly fabricated in-plane MNs based on inclined ultraviolet lithography and electroforming, and then converted these in-plane MNs into out-of-plane MN array by directly assembling the plane MN sheet one by one. Using this MN array, a negative mold was obtained by replicating it. Finally, the polycarbonate out-of-plane MN array was fabricated using the negative mold in a hot-embossing machine.
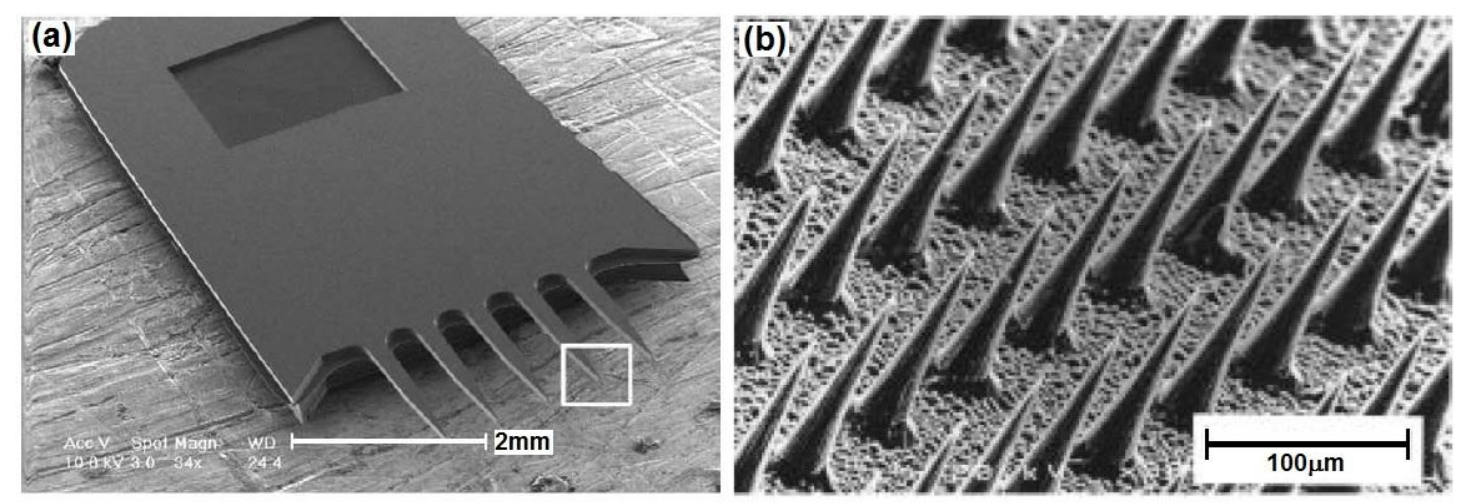

Fig. 2 SEM images of MNs. (a) A typical in-plane MN sheet made of silicon, Scale bar=2 mm [55]; (b) A typical out-of-plane MN array made of silicon, Scale bar=100 $\mu \mathrm{m}$ [9]. Image (a) and (b) are adapted from references [55] and [9], respectively.

\subsection{Categorized by drug transportation method}

MNs can also been categorized as solid MNs [9] and hollow MNs [60]. Just as the name implies, the former is solid, and the latter is hollow providing a special conduit for drug delivery into the skin. Usually, the two types of MNs have different drug transportation strategies. Therefore, based on the different drug transportation strategies, MNs can be categorized as: 'poke and patch', 'coat and poke', 'poke and release' and 'poke and flow'. The drug delivery strategies of the four types of MNs are described in a schematic as shown in Fig. 3. In the present review, we just simply introduce some basic knowledge about the four types of MNs and refer the readers to a comprehensive review by Kim et al. [11] for more details.

In 'poke and patch' manner, solid MNs are inserted into skin to form micro-scale pores in the skin surface. After removing the solid MNs, a drug patch is applied and the drug formulations diffuse slowly into the deep tissue through the residual pores. Of course, the solid MNs can also be used to scratch the skin to form strips of damages on the skin surface, and then the drugs can 
diffuse into the skin. The processing of this type of MNs is relatively simple. A great variety of materials, including silicon, ceramic, glass, metals and non-degradable polymers, are available for the fabrication of solid MNs. It has been proved that this method can significantly enhance the drug delivery efficiency [1,9], but the efficiency would be influenced by the shape, size, and density of the pores or scratches generated by the solid MNs.

As for 'coat and poke', the MNs are coated with water-soluble drug formulations. After the insertion of the drug-coated MNs into the skin, the coated drugs dissolve off the MNs and go into the skin, and then the MNs are pulled up. Matriano et al. [61] showed that using coating solid MNs with ovalbumin a 50-fold higher immune response was induced compared to intramuscular administration of the same dose in guinea pigs. Besides fabricating MNs themselves, appropriate coating technique must be employed for drug coating. This needs a more sophisticated design and fabrication technique than the 'poke and patch' MNs. Usually, the MNs are coated by dipping or spraying an aqueous solution of drugs. In order to improve the coating effect, some surfactants may be employed to enhance the wettability of the MN surface, or dip MNs into the coating solution repeatedly. Certainly, there are also some requirements on the coating formulations themselves, so some drug formulations cannot be applied in this manner.

About 'poke and release', MNs must be made from biodegradable or water-soluble materials, such as some biodegradable polymers and water-soluble sugars. The drugs are usually encapsulated within the MN matrix. After insertion into the skin, the MNs would completely degrade or dissolve in the skin, thereby the encapsulated drugs releasing is realized. In contrast to the former two types of MNs, there are no bio-hazardous sharp wastes to be disposed after use. Dissolving MNs are typically fabricated by micro-molding, but they have also been fabricated by drawing up liquid formulation to form tapered MNs at high enough temperature, and then solidified by cooling [50]. Ultrasonic welding approach has also been used with micro-molding to fabricate biodegradable MNs [62,63]. In this way, damage to encapsulate compounds was effectively controlled by welding polymer particles together without bulk heating of the polymer.

The 'poke and flow' is the way employed only by hollow MNs. Similar to hypodermic injection, the liquid drug formulations can flow into the skin through the conduit of MNs driven by pressure. Thereby, the drug flow rate can be deliberately controlled with the assistance of some flow-control equipments, such as syringe and micropump. Sometimes, hollow MNs can also been used as a channel for drug diffusing into the skin with a non-pressurized reservoir filled with drug. Over the three former types of solid MNs, the primary advantage of hollow MNs is that the liquid drug formulation can be directly delivered into the skin, so it is unnecessary to dry the drugs and also the damage induced by drying process can be avoided. Additionally, hollow MNs can be used for tissue sampling, which is significant in healthcare. However, because there are additional drug 
conduits in MNs, the manufacture may become more complicated than that of solid MNs. Methods including laser micromachining, deep reactive ion etching, deep X-ray photolithograhpy, wet chemical etching, are often used to fabricate hollow MNs [11]. Additionally, the hollow structure, usually a shell structure, may bring extra risks of buckling failure to hollow MNs [64]. It should be noted that, in the case of tissue sampling or signal monitoring, the length of MNs should be at least bigger than $1500 \mu \mathrm{m}$ [13], so that it can insert into the deep skin tissue. Undoubtedly, such high aspect ratio of MN means more difficulties in the fabrication and more probabilities of buckling. Recently, Li et al. [53], combining the drawing lithography, electroplating and laser cutting techniques, successfully fabricated an optimized hollow Nickel MNs with a length of 1800 $\mu \mathrm{m}$, an inner diameter of $60 \mu \mathrm{m}$, a tip diameter of $120 \mu \mathrm{m}$, and a $15^{\circ}$ bevel angle.

As mentioned above, the tiny size of $\mathrm{MN}$ is helpful for painlessness and minimal invasiveness, but it probably also induces mechanical failure during insertion of MN into the skin. In practice, no matter what a type of the MNs, the mechanical failure must be prevented in clinical applications. Unfortunately, this goal is not easily achieved because there are many factors to influence the mechanical performances of MNs. Therefore, studies on the mechanics problems with respect to MNs are reviewed in the following sections.

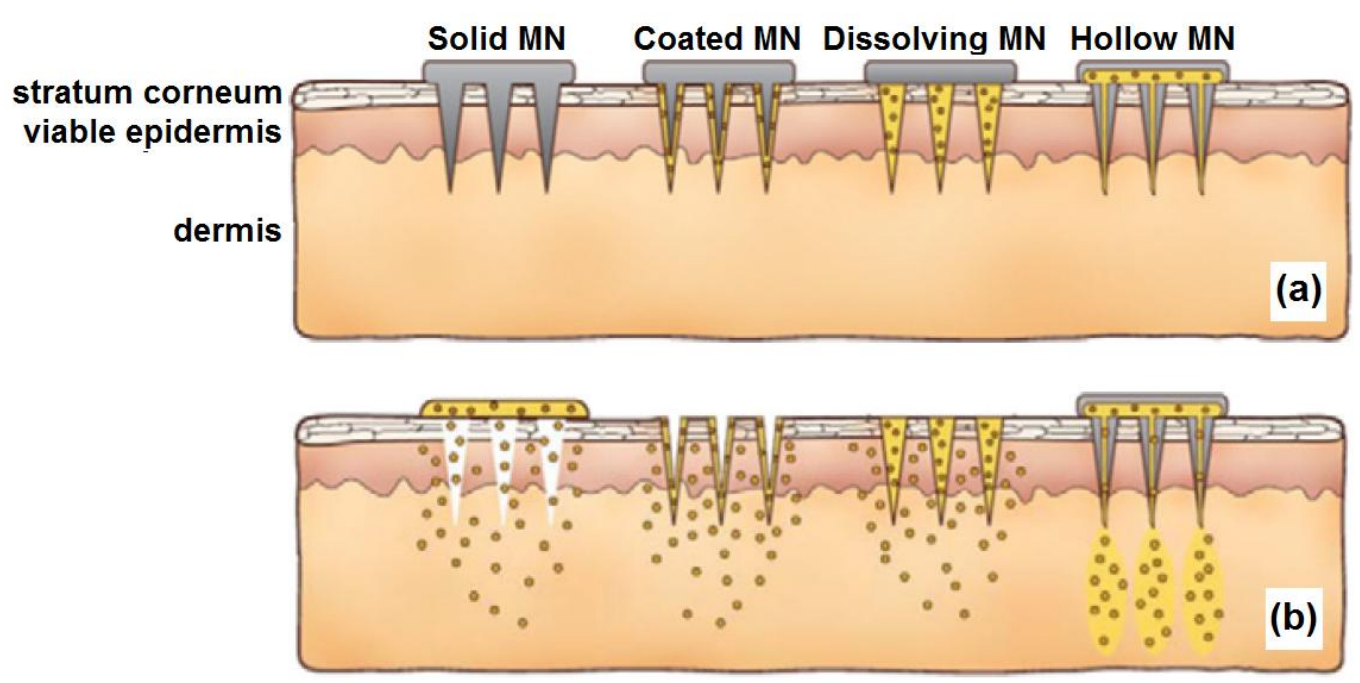

Fig. 3 Schematic diagrams showing the drug delivery strategies of different types of MNs. The microneedles are applied to the skin (a), and then used for drug delivery (b). Images are reproduced from Ref. [11].

\section{Insertion behavior of MNs into tissue}

Before the appearance of MNs, there have been a number of researches on the insertion behavior of hypodermic needle into skin or other tissues. In spite of the difference in size between the $\mathrm{MN}$ and hypodermic needle, these studies provide many valuable findings for people to understand the insertion behavior of MNs. Although it is not entirely clear how the pain is caused 
by needle insertion, it is usually believed that a small damage of skin tissue will reduce the possibility to cut the nerves, and a smaller resistance force of needle against the skin is also expected to reduce the compression force to the nerves near the needle tip. Therefore, a small size of MNs and a low inserting resistance force are always expected. As described by some researchers [1,64-68], the total resistance force acting on needle is consisted of three parts:

$$
f_{\text {needle }}(z)=f_{\text {stiffness }}(z)+f_{\text {friction }}(z)+f_{\text {cutting }}(z)
$$

where $f_{\text {needle }}$ is the total resistance force acting on the needle; $f_{\text {stiffness }}$ is the stiffness force induced by the elastic properties of the skin, so it is pre-puncture force; $f_{\text {friction }}$ is the friction force exerted by the surrounding tissue of the skin against the needle surface during its insertion process; $f_{\text {cutting }}$ is the cutting force that are necessary for slicing through the tissue. Obviously, $f_{\text {friction }}$ and $f_{\text {cutting }}$ belong to post-puncture force.

Simon and Okamura $[65,66]$ modeled the stiffness force using a nonlinear spring model:

$$
f_{\text {stiffness }}= \begin{cases}0 & z<z_{1} \\ a_{1} z+a_{2} z^{2} & z_{1} \leq z \leq z_{2} \\ 0 & z>z_{3}\end{cases}
$$

where $z$ is the needle tip position and $z_{1}, z_{2}$ and $z_{3}$ are described in Fig. 4. $a_{1}$ and $a_{2}$ are fitting constants based on experimental data. $f_{\text {friction }}$ and $f_{\text {friction }}$ are a little complex. Fortunately, because the length of $\mathrm{MN}$ is very small and it is usually to retain the $\mathrm{MN}$ tip in superficial layer for painlessness and minimal invasiveness, $f_{\text {friction }}$ and $f_{\text {friction }}$ are very small and can be simply ignored. However, it should be noted that, $f_{\text {friction }}$ and $f_{\text {friction }}$ cannot be ignored in evaluating the insertion behavior of long MNs for sampling. In that case, a much deep insertion is necessary.

In general, the insertion force, defined as the force required to puncture the top layer of the skin surface, is one of the most important parameters to evaluate the property of MNs. Based on Eq. (2), it can be found that the insertion force is actually the maximum stiffness force. Obviously, a low insertion force usually represents tiny pain and minimal invasiveness, thereby a low insertion force is always expected. Many researchers have made great efforts to investigate what can influence the insertion force of MNs. In 2004, Davis et al. [69] fabricated hollow MNs with different tip cross-sectional areas. Based on experimental and theoretical analysis, they found that the MN insertion force increases linearly with the needle tip full cross-sectional area. Roxhed et al. 
[70] fabricated a MNs array with ultra-sharp tips (tip radius is less than $100 \mathrm{~nm}$ ). Their experimental results show that the insertion force is less than $10 \mathrm{mN}$, the lowest value that artificial MNs can reach to date. By sharpening the needles at the tip but not over the entire length of the needle, Khanna et al. [71] also significantly reduced the insertion force. Aoyagi et al. [42] found that a sharper tip can induce more severely stress concentration and help the micro-needle pierce the skin more easily. These findings show that sharpening the MN tip can effectively reduce the insertion force.

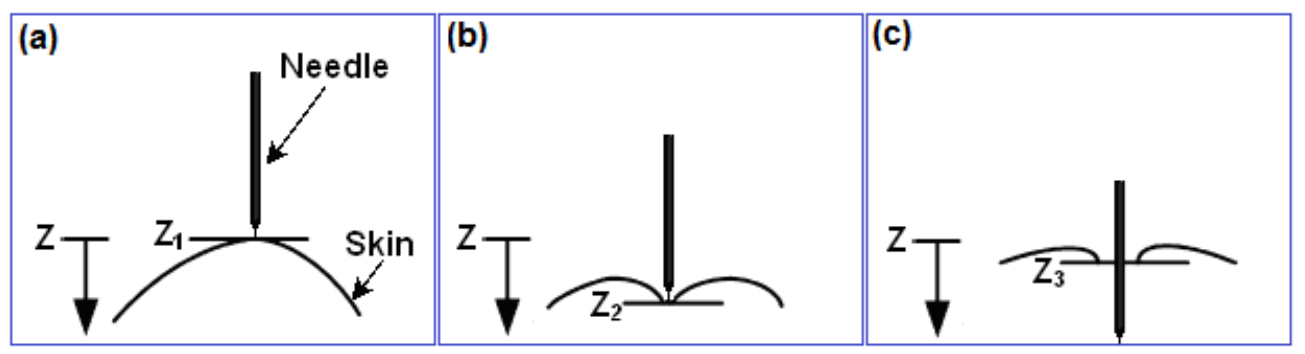

Fig. 4 The relationship between needle and skin at different insertion stages. (a) The needle begin to touch the skin surface without any interaction, i.e., a critical touch moment. (b) puncture. (c) post-puncture. Images are reproduced from Ref. [68].

As mentioned above, the insertion force can also be considered as the total resistance of tissue acting on the MN. For MN, this is external load, so low insertion force is helpful to decrease the possibility of mechanical failure of MN. For a hollow truncated cone needle as shown in Fig. 5a, if the wall is thin, the axial fracture force is modeled as $[69,72]$

$$
F_{f}=\pi D t \sigma_{b} \sin \alpha
$$

where $D$ is the outer diameter of the tip, $t=(D-d) / 2$ is the wall thickness, $\sigma_{b}$ is the ultimate strength of the needle material, and $\alpha$ is the tapering angle. If the $F_{f}$ is much bigger than the insertion force, the axial fracture can be usually prevented. It should be pointed out that the strength of human skin [73] is usually much smaller than that of most MN materials, a pure axial fracture would hardly happen, unless the MN wall is much thin. However, this does not mean that the occurrence of mechanical failure of MN can be completely eradicated. In many applications, besides axial force, lateral force component is probably inevitable during insertion process due to some causes, such as tilt insertion. In that case, bending stress may generate in the MN. Additionally, some MNs are high aspect ratio column-like structures, so buckling failure may happen. Therefore, the resistance of $\mathrm{MN}$ on bending failure or buckling failure must be investigated. For some MNs with simple profile, the bending stress and the critical buckling load 
can be theoretically predicted.

Consider the same hollow truncated cone MN as shown in Fig. 5a, the lower end is fixed and the upper end is free, a tilt concentrated force is applied in the free end. As shown in Fig. 5a, $L$ is the MN height, $\alpha$ is the tapering angle, $d$ is the inner diameter, $D$ is the outer diameter, $z$ is the coordinate along MN length. The wall thickness is a constant. The angle between the concentrated force, $F$, and the length axis is $\beta$. The maximum tensile stress at any cross section is obtained as

$$
\begin{aligned}
\sigma_{\mathrm{T}}= & \frac{32 F \sin \beta(L-z)}{\pi\left[\left(12 D \tan ^{2} \alpha-12 d \tan ^{2} \alpha\right)(L-z)^{2}+\left(6 D^{2} \tan \alpha-6 d^{2} \tan \alpha\right)(L-z)+D^{3}-d^{3}\right]} \\
& -\frac{4 F \cos \beta}{\pi\left[(4 D \tan \alpha-4 d \tan \alpha)(L-z)+D^{2}-d^{2}\right]}
\end{aligned}
$$

If the $\mathrm{MN}$ is a solid truncated cone as shown in Fig. 5b, we get the maximum tensile stress at any cross section as

$$
\begin{aligned}
\sigma_{\mathrm{T}}^{\prime}= & \frac{32 F \sin \beta(L-z)}{\pi\left[8 \tan ^{3} \alpha(L-z)^{3}+12 D \tan ^{2} \alpha(L-z)^{2}+6 D^{2} \tan \alpha(L-z)+D^{3}\right]} \\
& -\frac{4 F \cos \beta}{\pi\left[4 \tan ^{2} \alpha(L-z)^{2}+4 D \tan \alpha(L-z)+D^{2}\right]}
\end{aligned}
$$

In bending deformation, tensile stress, compressive stress and shear stress, all together may be generated, but tensile stress or shear stress due to the bending is usually the main factor that causes the $\mathrm{MN}$ fracture failure. This is especially the truth for some fragile materials, whose tensile strengths are always much smaller than their compressive strengths. Paik et al. [55] found that the out-of-plane bending force for silicon MN breakage is actually the smallest compared with any other applied forces. Therefore, the maximum tensile stress due to bending deformation should be smaller enough than the yield strength or fracture strength of the MN materials. Generally, the yield strength or fracture strength of materials are usually intrinsic properties independent of geometry, but, as indicated in Eq. (4) and Eq. (5), the tensile stress is considerably dependent on the geometry shape and size. Therefore, the geometry shape and size of MN should be elaborately designed. Furthermore, the material anisotropy may also need to be considered in some special case. Wilke et al. [74] investigated the mechanical failure characteristics of silicon MNs with lateral force and found that the silicon MNs will shear at the (111) crystal plane, which is known as the sliding plane with an angle of $54.74^{\circ}$. Moreover, it was found the shear strength is dependent on the location where the shear force is acting on.

Besides bending fracture, catastrophic buckling failure may take place during the insertion of MN into the skin. For a hollow truncated cone MN as shown in Fig. 5a, the critical buckling load is as following [75] 


$$
\begin{aligned}
P_{\mathrm{cr}}= & \frac{E}{80 \pi L^{2}}\left[\frac{5 \pi^{4}}{16}\left(D^{4}-d^{4}\right)+\left(5 \pi^{2}+\frac{5}{4} \pi^{4}\right)\left(D^{3}-d^{3}\right) L \tan \alpha\right. \\
& \left.+\left(15 \pi^{2}+\frac{5}{2} \pi^{4}\right)\left(D^{2}-d^{2}\right) L^{2} \tan ^{2} \alpha+\left(-120+30 \pi^{2}+\frac{5}{2} \pi^{4}\right)(D-d) L^{3} \tan ^{3} \alpha\right]
\end{aligned}
$$

For a solid truncated cone MN as shown in Fig. 5b, the critical buckling load is

$$
\begin{aligned}
P_{\mathrm{cr}}^{\prime}= & \frac{\pi^{2} E}{2 L^{3}}\left[\frac{\pi L D^{4}}{128}+\frac{\left(\pi^{2}-4\right) D^{3} L^{2} \tan \alpha}{32 \pi}+\frac{\left(\pi^{2}-6\right) D^{2} L^{3} \tan ^{2} \alpha}{16 \pi}\right. \\
& \left.+\frac{\left(\pi^{4}-12 \pi^{2}+48\right) D L^{4} \tan ^{3} \alpha}{16 \pi^{3}}+\frac{\left(\pi^{4}-20 \pi^{2}+120\right) L^{5} \tan ^{4} \alpha}{40 \pi^{3}}\right]
\end{aligned}
$$

where $E$ is the elastic modulus. Obviously, it can be found that soft materials with low elastic moduli are susceptible to buckling failure. Now let us investigate the influence of tip sharpening on the critical buckling load. If the height and base diameter are unchanged, sharpening the tip means a decrease in tip diameter, $D$, and an increase in include angle, $\alpha$. Although Eq. (6) and Eq. (7) are complicated, through several times of numerical calculating, one will find that the critical buckling load will decrease with tip sharpening. This tells us that it is inappropriate to blindly decrease the insertion force only by sharpening the MN tip. Chandrasekaran \& Frazier [76] studied the truncated pyramid-like MNs and found that if the tip is too sharp, fracture failure is unavoidable. In other word, low insertion force cannot always assure the mechanical safety of MNs. Paik et al. [55] studied four types of MNs as shown in Tab. 1. Experimental results show that the average insertion force into chicken breast flesh for MNs of type A, B, C and D are 65.7, 167,103 , and $80.9 \mathrm{mN}$, respectively. They pointed out that a small tip angle can effectively reduce the insertion force, but it may result in severe tip damage before the insertion of $\mathrm{MN}$ into the tissue, so a trade-off between sharpness of the tip and mechanical safety should be taken into account. From this point of view, they concluded that type D is an optimal shape design. Usually, the safety factor, defined as the ratio of failure force (fracture force or critical buckling load) to insertion force, is a more effective parameter to judge the mechanical safety of MNs.

With the knowledge given by Eq. (4) to Eq. (7), it is not hard to see that a tall MN is always susceptible to bending failure or buckling failure. In other word, shortening the MN is an option to decrease the possibility of mechanical failure. However, because of the elasticity of skin, considerable displacement will produce before the insertion of MN into the skin. If we defined the displacement at the puncture point as insertion displacement, the minimum length of MNs should be longer than this value. Therefore, it is essential to decrease the insertion displacement so that a short MN can pierce the skin. It is notable that short MNs can pierce the skin successfully by a vibrating method [77,78]. Verbaan et al. [79] pierce the skin successfully using short MNs when the MN array was moved in a high-speed impacting manner. Aoyagi et al. [42] found that the 
insertion displacement can be reduced by stretching the tissue or vibrating the MNs. As determined by Kong \& Wu [80] the structure and mechanical properties of skin can also influence the insertion behavior of MNs into skin. Recently, a 70 80 $\mu \mathrm{m}$ long MN was successfully inserted into the skin by scratching the skin before insertion [81]. It should be noted that the length of MNs must be larger than the insertion displacement is only a basic requirement. In MN designs, other factors must be simultaneously taken into account. For example, the influences of MN length on drug delivery efficiency, pain, etc. $[82,83]$ must be carefully studied. Hence, in the following section, we will review some studies on drug delivery effect and insertion pain with respect to MNs.
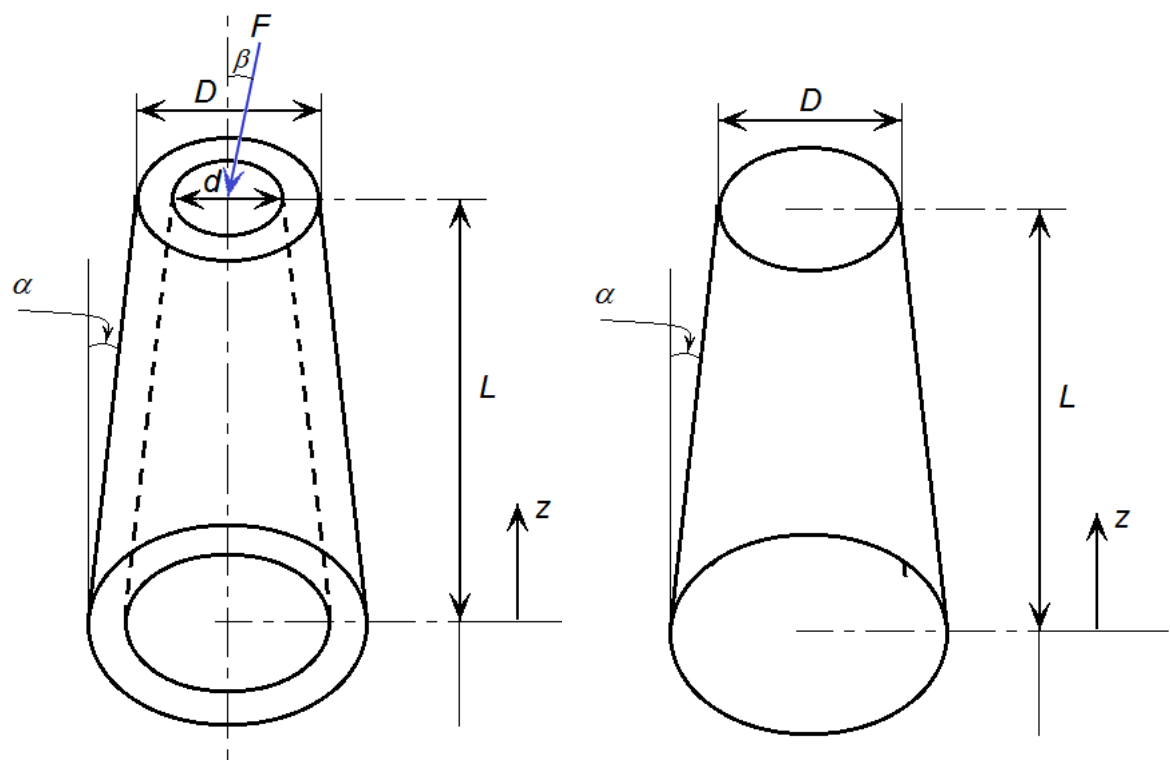

Fig. 5 Schematic diagrams of a hollow truncated cone microneedle and a solid truncated cone microneedle;

Tab. 1 Four types of microneedles studied by Paik, et al. Experimental data show that D-type microneedle has optimal properties. Table is reproduced from Ref. [55].

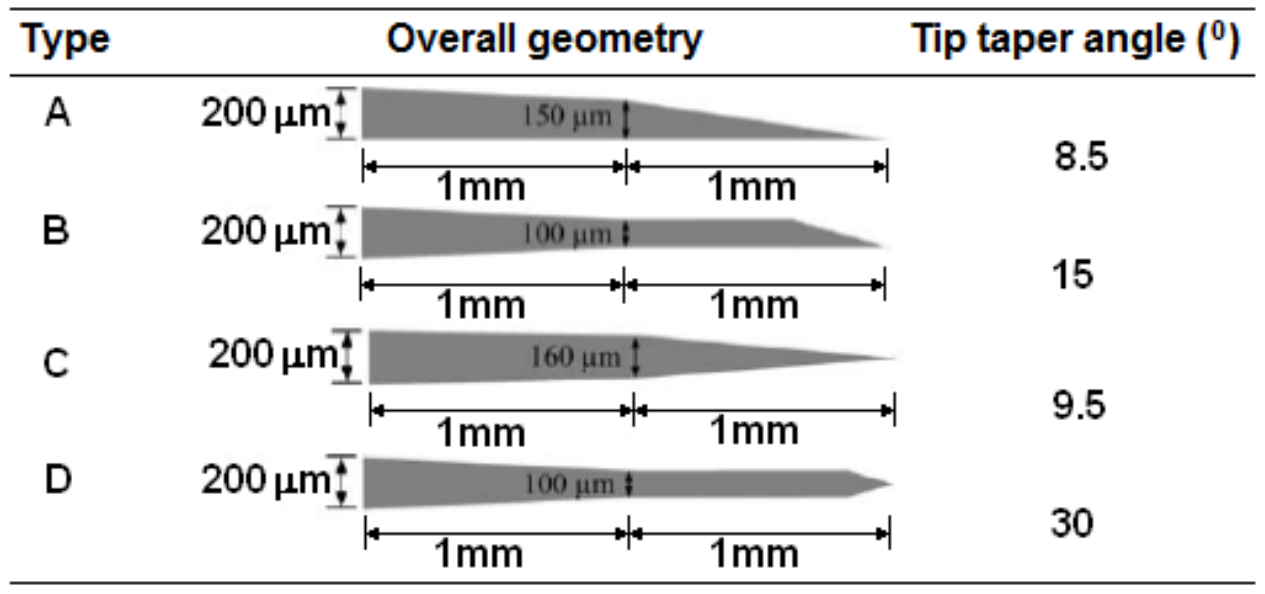




\section{Drug delivery efficiency and pain of MNs}

The final goal of using MNs is to delivery drugs into the skin efficiently and painlessly, so it is necessary to investigate the influence of MNs on these problems. For solid MNs, it was found that the MNs with flat tips are better for enhancing skin permeability than those with sharp tips, as well as prolonging the detained time in the skin can also enhance the skin permeability [81]. Moreover, increasing the pressing force will also enhance the skin permeability. Badran et al. [37] found that the longer the MNs, the better the enhancement in skin permeability. They proposed that this is because the longer MNs penetrate the SC and enter the underlying dermal tissue resulting in an increased hydration of the SC. Gomaa et al. [84] also found that longer MNs can enhance the skin permeability until a certain MN length threshold was reached. They attribute this phenomenon to a skin compaction and dehydration effect. They also found that increasing the number of the MNs in the array produces a similar pattern to that of increasing MN length. However, if the MN density is too high, individual MN may no longer disrupt the SC barrier due to a 'bed of nails effect'. A better effect may be achieved using a MN array with lower needle density and longer needle length [56]. Bal et al. [85] pointed out that the MN geometry, but not the insertion speed affects the shape and depth of the conduits, so the MN geometry plays an important role on drug delivery efficiency. Watanabe et al. [86], however, found that the skin permeability can be enhanced by increasing the impact velocity of MNs. The causes of this contradiction are still not clear. It is interesting to note that Cai et al. [33] fabricated a MN array out of bioceramic with flexible and self-swelling substrate. Experimental results show that such a MN array can make drug release faster than the MN array with a rigid substrate. More recently, a type of dissolvable MNs mounted on the solid MNs was manufactured [87]. It was found that, compared with conventional dissolvable MNs, these separating MNs can dramatically enhance the drug release rate.

For hollow MNs, the drug formulations are usually fluidic transported through the conduits in MNs, so the drug delivery rule is different with that in solid MNs. As the inner conduits of most hollow MNs are usually tube-like, the flow resistance can be modeled by a laminar Poiseuille's Law as [88]

$$
R=\frac{\Delta P}{Q}=\frac{128 \mu L}{\pi D^{4}}
$$

where $R$ is the hydraulic resistance, $\Delta P$ is the pressure difference, $Q$ is the volumetric flow rate, $D$ is the diameter of pipe, $\mu$ is the dynamic viscosity and $L$ is the length of pipe. This prediction is experimentally validated [89], in which the flow rate increases approximately linearly with the increasing driven pressure. However, Eq. (8) describes only the relationship between intrinsic flow resistance and geometry parameters of hollow MN without the presence of 
skin. Indeed, the flow rate of hollow MN is also influenced by the skin and other factors. Because the skin tissues provide extra flow resistance, the flow rate will dramatically decrease compared with that in a MN without the presence of skin [31]. Wang et al. [77] found that the flow rate can be significantly increased by withdrawing or vibrating the MNs. They attribute this to the fact that the dermal tissue might have been penetrated into the needle bore owing to tissue coring or that the flow might be hindered by the dense dermal tissue surrounding the needle tip. It was revealed that compaction of skin probably press out most water and thereby significantly reduce the flow conductivity of skin beneath the MN tip [30,31]. Retraction of MN can relieve the skin compaction and increase the flow conductivity. Furthermore, it was found that a bevel tip is helpful to weaken the compaction of tissue beneath the needle tip, so the infusion flow rate can be increased by using a bevel tip. These observed phenomena are very different from those in solid MN [81]. Moreover, it was found that the MN tip opening size has no significant effect on infusion flow rate [30]. This is also because that the resistance induced by the dense dermal tissue beneath the tip play a dominant role on the resistance to flow when compared to the resistance offered by the conduit of MN itself. Besides improving the MN design and the manner of application, the hyaluronidase, an enzyme that degrades hyaluronic acid in the extracellular matrix of the skin, can be co-injected with the drug formulations to reduce the fluid flow resistance [30, 90]. Recently, Le-Thanh et al. [91] proposed a novel hollow blood collection MN design, in which the diameter of the conduit near the tip is larger than the diameter of the conduit in the body part. Numerical analysis shows that this design endows the MN with combination of high mechanical strength, high extraction efficiency and minimal clogging problem for high-volume blood collection. Finally, it should be pointed out that the flow rate usually increases with deeper insertion [31], but the intradermal immunogenicity may not be affected by injection depth [92].

As an attractive alternative to hypodermic injection, one of the most important advantages of $\mathrm{MN}$ is the painlessness, but only a limited number of studies are associated with this topic. To our knowledge, Kaushik et al. [10] are the first to study the pain caused by MN insertion. Experiments show that the pain caused by pressing an array of 400 silicon MNs into the human forearm skin is much less than that caused by a 26-gauge hypodermic needle. More detailed experiments carried out by Gill et al. [93] show that the MN length has the strongest influence on pain, where a 3-fold increase in MN length increases 7-fold in pain. Other studies [94,95] show that the insertion of MN shorter than $400 \mu \mathrm{m}$ is painless. In addition, Gill et al. [93] found that the number of MNs also affected the pain, where a 10 -fold increase in the number of MNs increases pain by just over 2-fold. Compared with MN length, the MN tip angle, thickness and width have weak effects on pain $[93,96]$.

For hollow MNs, pain is affected not only by the geometry size of MN, but also by the flow of 
liquid drug solution. Gupta et al. [90] found that application of larger pressure causes more pain, and $\mathrm{MN}$ retraction also increases pain, but lower flow rate and co-injection of hyaluronidase would lower pain. Gupta et al. [97-99] and Van Damme et al. [100] further studied the pain caused by hollow MNs while delivering different types of liquid drugs at different transportation rate. They found that painlessness is usually associated with MNs. Consider these findings, we believe that pain is not an important problem in $\mathrm{MN}$ designs.

From the literatures discussed in the above segments, it is easily to find that the optimization of MN is actually a compromise between different factors. This is obviously a hard work. Fortunately, there are some bio-microneedles in nature, which exhibit excellent integrated properties. Study and learn from them, we may get some inspirations to improve the MN design. In the following section, we will review some studies on bio-microneedles.

\section{Bio-microneedles}

Mouthparts are the feeding apparatus of insects, which have been modified in various feeding structures to perform the ingestion of a variety of food in different feeding manner. The earliest insects had chewing mouthparts. With the evolution, there have developed other types of mouthpart including piercing-sucking mouthparts, sponging mouthparts, siphoning mouthparts, grasping-sucking mouthparts, rasping-mouthparts, scratching mouthparts, and chewing-lapping mouthparts. Among these species, insects with piercing-sucking mouthparts do not chew food, but have a needle-like structure allowing them to pierce plant or animal tissue and sucking liquid from the host organisms. The most familiar insects with piercing-sucking mouthparts, undoubtedly, are mosquitoes. From early time the mosquitoes have received considerable attentions since they are important vectors of a wide range of viral and parasitic diseases affecting both human and animal [101]. It is well known that the mosquitoes can pierce human skin and suck blood in a much stealthy manner, meanwhile, without any mechanical failure. The length of the mosquito mouthpart is totally about 2 2.5 millimeters depending on species. How can such slender stylets penetrate animal' or human' skin? We will firstly seek the answer from the specialized structures of the mosquito's mouthpart.

The female mosquito mouthpart (called a proboscis) is very specialized and adapted to pierce skin and then suck blood. Externally, the visible part of the proboscis is the labium, whose surface is fully covered with scales and there are a pair of labellar lobes on the front tip. Detailed anatomy studies show that, besides the labium, mosquito proboscis consists of two mandibles, two maxillae, one labrum, one hypopharynx and one labium. When the mosquito is at rest, the other components of the mouthpart are fully enveloped in the labium sheath [101-105]. The paired mandibles are modified to thin and sharply pointed. The paired maxillae are also thin and sharp. SEM 
observations show that, besides an ultra-sharp tip, there are saw toothed microstructures along the two sides of the maxilla near the tip as shown in Fig. 6 [106]. The paired maxillae are actually the main organs for piercing the host's skin [104]. Having such an ultra-sharp tip is beneficial to reduce the insertion force. The mandibles and maxillae are reduced or absent in all males and some females who only feed on plant fluids. The labrum, forming an entirely enclosed tube with a side open tip, is the largest stylet used for piercing and then sucking blood from the skin [107]. The food is taken through the tube formed by the labrum anteriorly and the mandibles posteriorly. The hypopharynx is stylet-like and contains a duct, through which the saliva, as an anticoagulant, is injected. It should be noted that, as shown in Fig. 6a, the labrum has a reinforcing-rib-like bicuspid tip instead of the common unicuspid tip on other mouthpart components. Although the comprehensive effects of reinforcing-rib-like microstructure are not clear, it is obviously beneficial to prevent topical mechanical failure.

As mentioned above, the length of the mosquito mouthpart is totally about 2 2.5 millimeters, and the typical outer diameter of the mouthpart is only about 15 25 $\mu \mathrm{m}$. Can only these complex but delicate structures make sure such slender stylets to pierce the animals' or humans' skins without mechanical failure? Unfortunately, the answer is no. In fact, if we directly press the mosquito's mouthpart against the skin, it would never successfully pierce the skin. This means that there are other mechanisms to help the mosquitoes pierce the skin.

By means of some special technologies, the biting behaviors of living mosquitoes have been carefully studied. In 1939, Gordon \& Lumsden [108] studied the biting behavior of mosquito when it was piercing and sucking blood from a living tissue, the web of a frog. More recently, by means of the high-speed video camera, many researchers $[42,106,107,109,110]$ restudied this topic more precisely. It was found that the mosquito hardly pierces the skin directly. In fact, it will repeatedly probe the skin until it finds a suitable spot [110]. Once the suitable spot is determined, the labella of the labium will firstly anchor down into the skin surface and then begins to axially press the proboscis while pushing it into the skin [110]. Moreover, Aoyagi et al. [42] speculated that the labium may give a tension to the skin surface by opening the paired labella for easy insertion. At this moment, no visible bending deformation occurs on the labium. After that the mosquito will move its head up and down slightly, and then the fascicle is driven deeper into the skin. A distinct feature is that the labium increasingly bends backward as watching in side-view, i.e., a labium loop forms near the head. As described by Ramasubramanian et al. [110], the labium loop appears and disappears at about a constant frequency $(15 \mathrm{~Hz})$ during the early penetration stage. As the penetration depth increases, the labium loop increases. At the same time, the oscillation frequency of mosquito's head decreases form the initial $15-17 \mathrm{~Hz}$ to about $6 \mathrm{~Hz}$. Kong [106] gave more detailed data on the relationship between oscillation frequency and time. It was 
found that the oscillation frequency of mosquito's head decreases monotonically with time from the initial $10-15 \mathrm{~Hz}$ to $3-6 \mathrm{~Hz}$ and the average penetration process usually lasts for about 10 15 seconds.

As described above, there are many saw toothed microstructures distributed on the paired maxillae. The vibration of the mosquito's head makes the paired maxillae become exactly a pair of micro-saw. This is undoubtedly beneficial to cutting the skin tissue and reducing the insertion force. Moreover, the saw-like shape may decrease the contact area between the surface of the stylets and the skin tissue. For this reason, the frictional force reduction can be effectively reduced [42,111]. A deliberately designed experiment was carried out by Kong \& Wu [107]. It was found that the insertion force of a living mosquito's fascicle (Aedes albopictus) into a human skin is only about $16.5 \mu \mathrm{N}$, which is about three orders of magnitude smaller than the lowest known insertion force for an ultra-sharp silicon MN [70]. Besides lowering the insertion force, the vibration may also provide some mechanisms to increase the resistance of the fascicle against the buckling failure. It was hypothesized that the mosquito may apply some non-conservative Beck forces on the fascicle during its insertion process by moving the fascicle back and forth [110]. Theoretical analysis shows that combination of the non-conservative force and the additional lateral support provided by the labium can increase the critical buckling load of the mosquito's fascicle by a factor of 6. Furthermore, it was also found that the mosquito will reduce the applied force, if it senses the buckling initiation, and then it will reorient and reposition the head slightly and attempt again [110]. In other words, mosquitoes have consciousness to avoid buckling failure of fascicle.

Considering the characteristics of mosquitoes described above, it is easily to find that the excellent properties of the mosquito's needle-like proboscis benefit from the combination of elegant microstructures and intelligent insertion modes. In nature, there are many other similar samples such as the mouthpart of tsetse fly [112,113], mouthpart of cicadellidae [114], stings of honey bees and paper-wasps [115], and so on. In addition, there are also some special bio-microneedles which possess excellent performance mainly due to the optimal geometry, for example, the caterpillar spine [116]. 

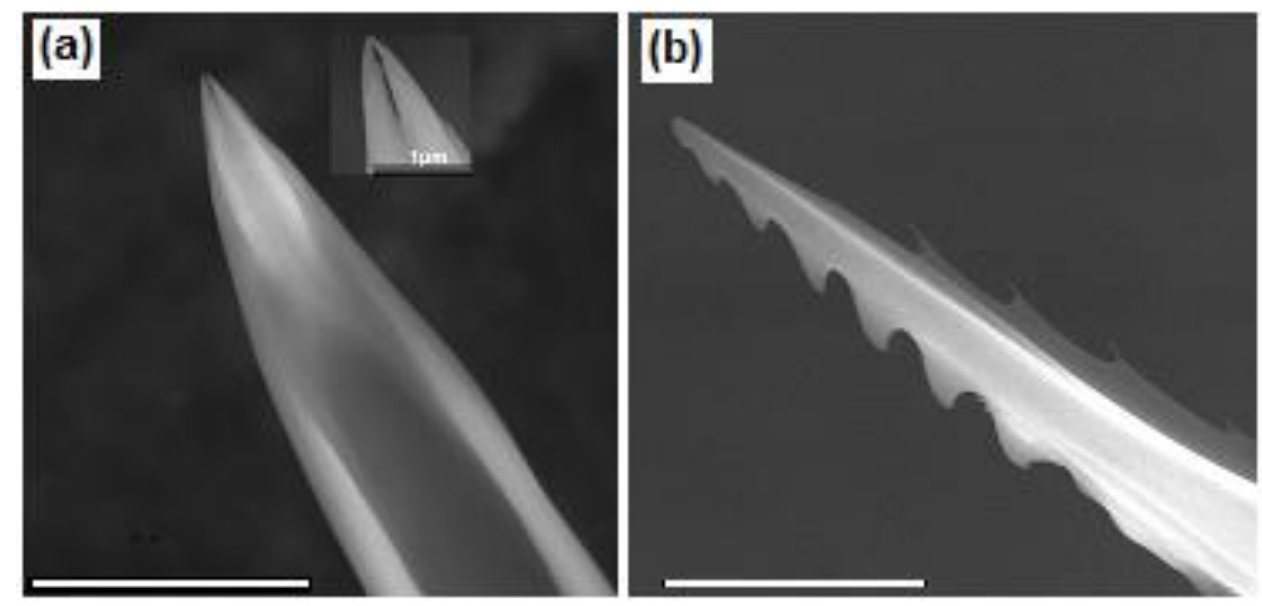

Fig. 6 SEM images of mosquito's fascicle. (a) Labrum with a fork-like tip and two reinforcing ribs. Scale bar=30 $\mu \mathrm{m}$. (b) Maxilla with micro/nano-saw teeth at the two sides. Scale bar=10 $\mu \mathrm{m}$. Images are reproduced from Ref. [106].

Ma et al. [116] firstly studied the insertion behavior of the spine of caterpillar, Parasa Consocia. Taking advantage of the SEM and microcomputed tomography system, it was found that this kind of spine is a hollow tapered needle with a side-open tip. They measured the insertion force of caterpillar spine into fresh mouse skin. It was found that the average insertion force is only about $173 \mu \mathrm{N}$, which is higher than that of mosquito's fascicle into human skin [107], but is still two orders of magnitude lower than that of the artificial ultra-sharp silicon MN [70]. The compressive and bending fracture forces were also deliberately measured, respectively. The average compressive fracture force is about $2.98 \mathrm{mN}$ and the average bending fracture force is about 2.27 $\mathrm{mN}$, respectively. This represents that the caterpillar spine has a high safety ratio over 13. Theoretical analysis shows that the caterpillar spine' shape is similar with an equal-strength-beam as shown in Fig. 7, i.e., an optimal shape for resisting to bending fracture and compressive buckling failure [117], the two main typical failure modes in MN applications. Further measurement by using an atomic force microscopy shows that, the Young's modulus and hardness of the caterpillar spine exhibit a considerable gradient as shown in Fig. 8, i.e., the values of Young's modulus and hardness increase are higher near the tip. A tip with relative high modulus and hardness is apparently helpful for the insertion. It should be pointed out that these values were all obtained through quasi-static loading tests, i.e., both the low insertion force and high resistance against mechanical failure benefit from only its geometry size and shape, and materials properties. This situation is considerably different from that of the mosquito's mouthpart. Obviously, this means more conveniences in mimicking design of MNs, especially the hollow MN design. Additionally, the caterpillar spine also has some other features, including barbs distributed over the surface, neck-like groove near the tip, etc., which, perhaps, are not inappropriate for TDD MN, 
but are consistent with its defense/attack function. In other word, Nature is exactly a genius.
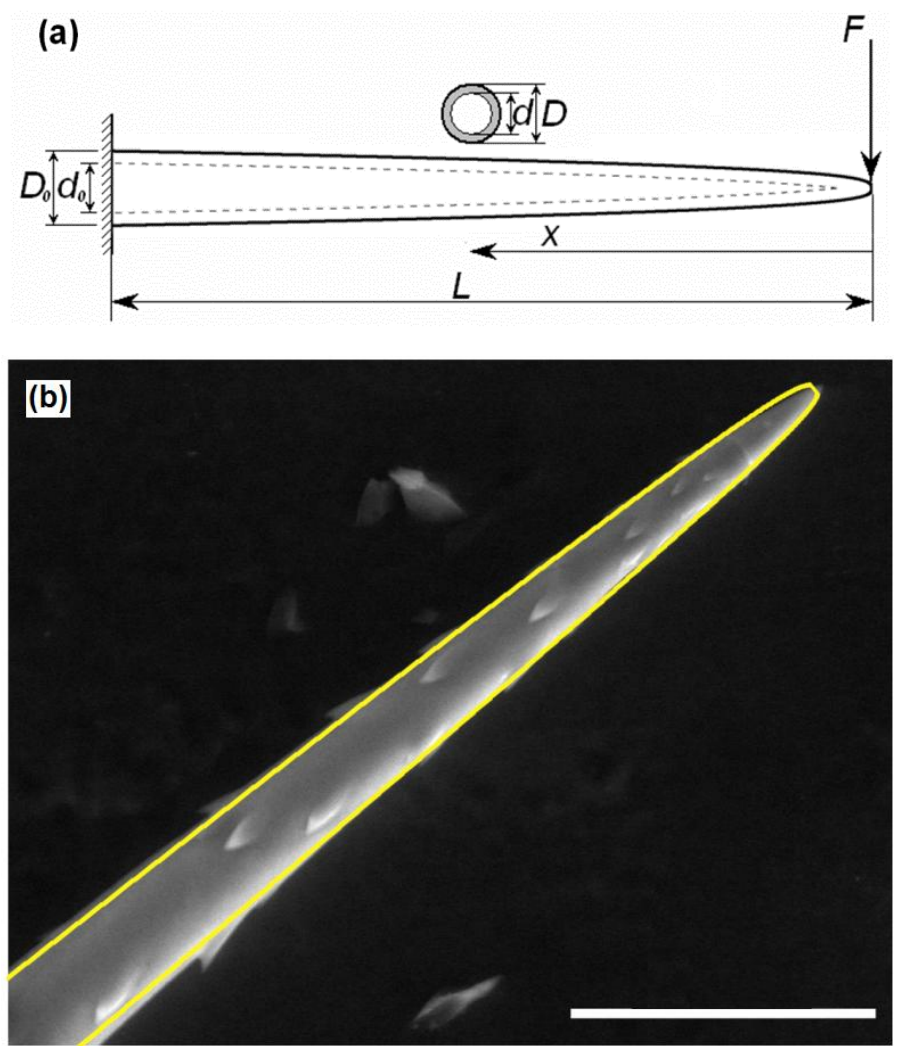

Fig. 7 (a) The shape of an equal strength beam with hollow circular cross section and constant wall thickness. The inner profile is depicted by the dotted lines, (b) Comparison of the equal strength beam (yellow) and the Parasa Conscocia caterpillar spine. Scale bar is $50 \mu \mathrm{m}$. Images are reproduced from Ref. [116].

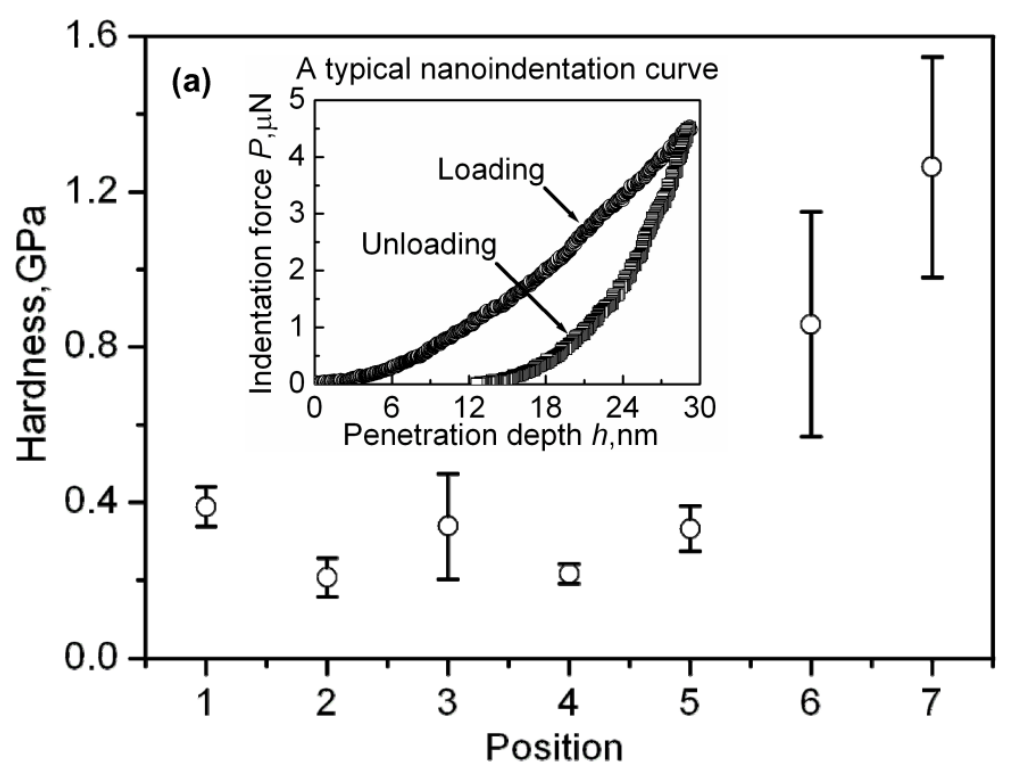




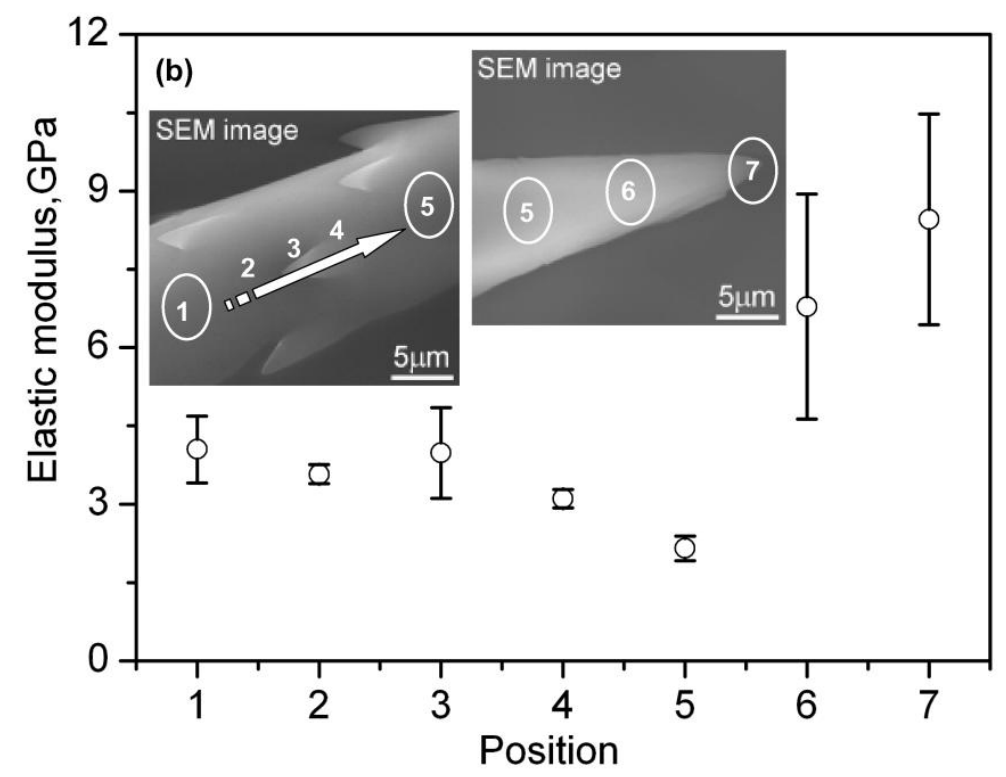

Fig. 8 Distributions of (a) hardness, and (b) elastic modulus on the Parasa Consocia caterpillar spine surface along the length. The inset in (a) is a typical nanoindentation curve and the insets in (b) are the SEM images of a caterpillar spine with the locations of nanoindentation marked. It can be seen that both the hardness and the modulus near the tip are higher than those at the base part of the spine. Images are reproduced from Ref. [116]

\section{Bio-inspired MNs}

Inspired by the natural bio-microneedles, especially the mosquito's mouthpart, scientists have already made great efforts to manufacture bio-mimicking MNs. To our knowledge, Oka et al. [111] are the first group to manufacture a hollow silicon MN with a jagged shape inspired from mosquito proboscis. In their article, the fracture features were investigated by experiment and numerical simulation, as well as, the insertion force was also experimentally studied. Subsequently, Aoyagi et al. [42] further fabricated a type of MN with jagged shape made of biodegradable polymer. Experiments showed that the insertion force of this type of MN can be significantly reduced by vibrating the MN. This experimental design is inspired from the insertion mode of mosquito. Additionally, they mimicked the mosquito by stretching the silicone film and experiments showed that the insertion force can also be considerably reduced. Finally, inspired from the mosquito's proboscis, they analyzed the mechanism by which the combined needles comprising one central straight needle and two outer jagged needles, can insert into the skin tissue more easily. Finite element method (FEM) simulation results revealed that, by moving the three needles alternatively, i.e., vibrating the combined needles, the stress distribution can be confined in the space between two maxillae, which bring a merit to increasing the degree of the stress concentration at the tip area of the labrum. As a result, the insertion force was considerably reduced. A similar conclusion was also been drawn by Cho et al. [118], who studied the North 
American porcupine quill, a needle-like structure with barbs, and found that the distributed barbs can increase the stress concentration and reduce the insertion force. Recently, a real combined silicon MN system (see the inset of Fig. 9) mimicking mosquito's proboscis was successfully fabricated using electrochemically etch method [109]. The movements of the central straight needle and two outer jagged needles are controlled by PZT actuators independently. In their work, three inserting modes are investigated. Experiments showed that the insertion forces can be actually influenced by the moving modes of the three needles. The detailed relationship between the insertion forces and the moving modes is given in Fig. 9, in which it can be found that mode $\mathrm{C}$ produces the lowest insertion force. In mode $\mathrm{C}$, the central needle was moved firstly, and then the outer two jagged needles are simultaneously moved with $180^{\circ}$ phase delay to the central needle, which is similar with mosquito's inserting behavior [42]. These movements are repeatedly processed at $30 \mathrm{~Hz}$.

Yang \& Zahn [119] fabricated a hollow MN using a two-wafer polysilicon micromolding process. Through vibrating the $\mathrm{MN}$ in the $\mathrm{kHz}$ range using a vibratory actuator, the insertion force of the $\mathrm{MN}$ into an animal skin is reduced by more than 70 percent compared with quasi-static insertion. The authors thought there are three main mechanisms: (1) high-speed impact at high frequencies leads to an increase in the skin's dynamic stiffness, (2) cavitation induced by the interplay of vibratory pressure waves and the tissue fluid destroys tissue, and (3) localized thermal damage led by the frictional interactions between the $\mathrm{MN}$ and skin. Kong [106] was also inspired by mosquito's proboscis and successfully fabricated lancets with different types of micro-sawteeth on the blades (as shown in Fig. 10). Experiments show that the force required to cutting pig skin can be dramatically reduced by 98 percent when the jagged-blade lancet is moved coupling with a 15 $\mathrm{Hz}$ vibration. In contrast, if it is a common lancet without saw teeth, the cutting force can only be reduced by 30 percent even if applying a $15 \mathrm{~Hz}$ vibration.

A research group at Jilin University has made great efforts to study bionic hypodermic needles [120-122]. For example, Wang [122] was inspired by mosquito's mouthpart, and then fabricated hypodermic needles with non-smooth surfaces. By optimizing the surface parameters including distance, depth, width, etc., the drag force can be reduced by about 50 percent. It should be pointed out that insertions in their studies are quasi-static, i.e., no vibration exists. In their series works [120-122], they found that the drag-reduction attributes mainly to the reduction of contact surface, conversion of sliding friction to rolling friction, what are induced by the non-smooth surface. A mimetic needle inspired by North American porcupine quill was also successfully fabricated by Cho et al. [118]. Experiment show that this biomimetic needle can considerably reduce the insertion force.

From these works associated with bio-inspired needles, we can find that both the vibration and 
microstructures can effectively influence the insertion force, but the combination of them can achieve a best effect on insertion force reduction.

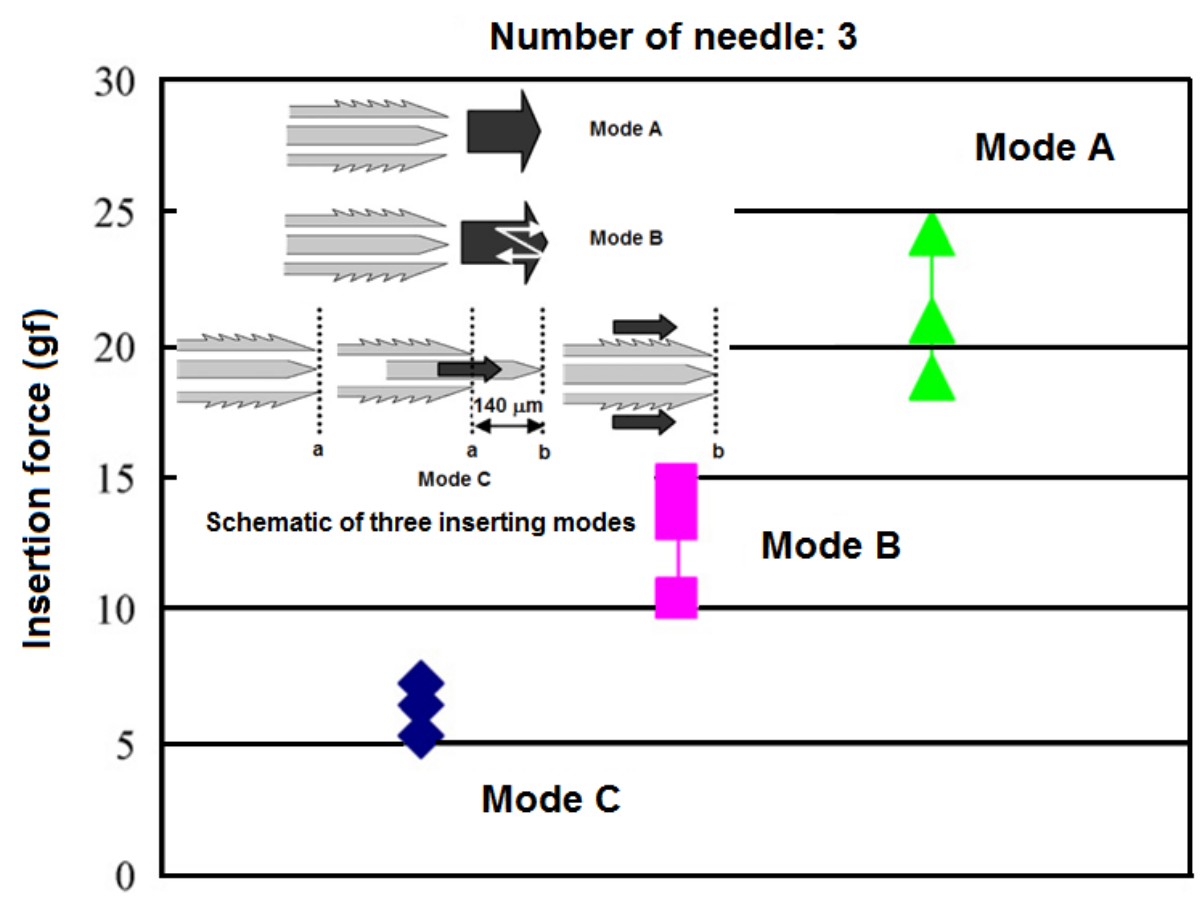

Fig. 9 Relationship between insertion force and inserting mode. The insert shows how the combined MN system with three MNs imitating the mosquito's fascicle to insert skin using different modes. Image is adapted from Ref. [109].
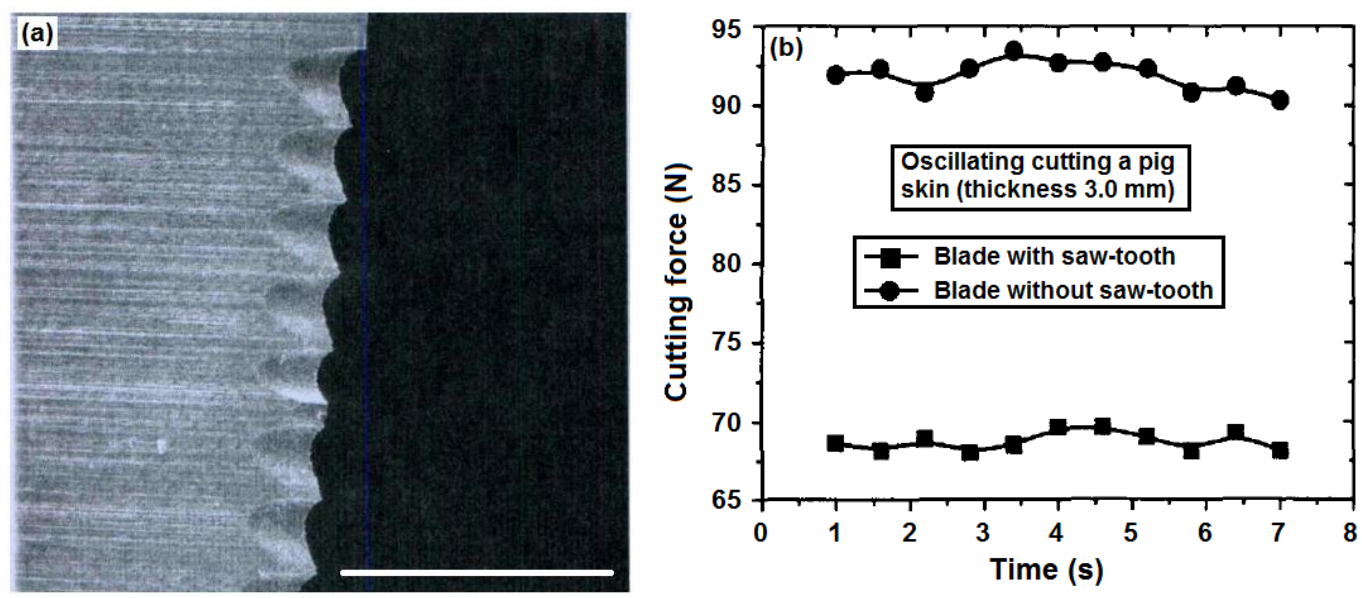

Fig. 10 (a) A SEM picture of a lancet blade with micro-sawteeth inspired by the mosquito's maxilla. (scale bar is $500 \mu \mathrm{m})$; (b) The cutting forces of lancet with/without micro-saw-tooth to cut a pig skin. Experiments show that the force required to cutting pig skin using this jagged-blade lancet can be dramatically reduced by 98 percent when it is moved up and down at $15 \mathrm{~Hz}$. In contrast, if the cutting force for a common lancet with smooth blade to cut pig skin can only be reduced by 30 percent even if applying a $15 \mathrm{~Hz}$ vibration. Images are reproduced from Ref. [106]. 


\section{Perspectives and conclusions}

Since its appearance in 1976, the MN technology has grown over four decades. To date, with the development of contemporary microfabrication technologies, a wide range of MNs have been successfully fabricated out of various materials including metals, silicon, glass, ceramics, and polymers. Among these materials, polymers, especially some biodegradable or dissolvable polymers, are considered as the most promising materials for $\mathrm{MN}$ fabrication due to their favorable biocompatibility [44]. Silicon has also received considerable attentions owing to the advanced silicon processing technologies. Metals are adopted to manufacture MNs due to their good combination of mechanical strength and toughness. These MNs, however, also have some disadvantages to some extent. For example, metal MNs produce sharp bio-hazardous tip wastes; silicon MNs are prone to brittle fracture during their insertion; polymers are usually soft and susceptible to buckling failure. The mechanical failures are always undesirable in the clinical applications. Fortunately, many research groups have made great efforts to study the corresponding mechanics problems of MNs, including the insertion force, critical buckling load, compressive fracture load, bending strength, and flow rate. Undoubtedly, fully understanding the mechanical behavior of MNs against skin or other tissues is still a key to improve the MN designs. Moreover, scientists also paid attentions to some bio-microneedles and revealed that these bio-microneedles have unique microstructures and intelligent strategies to enable them to pierce skin easily. Inspired by these bio-microneedles, mimic MNs have been successfully manufactured and proved to be very good for piercing/cutting the skin with a much low force.

Although MNs were originally developed for drug or vaccine delivery to the skin, to date other applications of MNs have also been investigated. For example, MNs are used to deliver drugs through sclera/cornea of the eye to treat posterior segment diseases of eye [16,123-126]. This technology can overcome the disadvantages associated with conventional delivery using topical eye drop or other side effects using systemic delivery $[16,123]$. MNs have also been explored as a combined tool with gene gun to reduce the operating pressure and then to reduce the damage of tissue/cell [17]. Long MNs were also used to record the signals of brain neurons [15]. Compared with electroencephalograms or electrocorticograms, this MN-assisted signal monitoring method can provide much high spatial resolution and obtain signals in the deeper brain tissue. Recently, nanoneedles, the extensions of MNs, have also been studied as biosensors, bio-signal monitor, membrane-penetrating delivery systems, and bio-imaging agents [18,19,127-131], for single cell. Perhaps, in the future, MN technology may make it possible to develop an intelligent system which can automatically sample, analyze, diagnose, deliver drug, and record signal, by combining with other micro-electromechanical systems, micro-fluidic chip system, remote diagnosis technology, remote signal monitoring technology, and robotic drug administrating technology. In a 
word, the great further growth of MNs is anticipated. This goal, however, is not easy to achieve. To achieve this goal, besides these technologies themselves, the corresponding mechanics problems associated with MNs are needed to further study. In practice, no mechanical failure, high delivery efficiency, painlessness, and minimal invasiveness, are basic requirements for $\mathrm{MN}$ and its corresponding technologies. In many practical applications, such as drug delivery on eye, brain neural signal recording, remote robotic surgery/therapy, and so on, it is necessary to understand the mechanics behavior of MNs against skin or other tissues in more details, so that the behavior of MNs can be precisely controlled and unexpected damages of other tissues caused by MNs insertion/cutting can be effectively avoided. The present corresponding studies are not enough to dissolve these problems. Even in the existing researches, there are still some contradictory findings. For example, Frick et al. [132] found that the insertion force of needle increases with increasing pre-tension in the skin. On the contrary, Figge \& Barnet [133] found that the force required to puncture a preloaded skin is less than loose skin. Aoyagi et al. [42] also demonstrated that stretching skin is useful to reduce the insertion force. Besides these contradictory results, there are also some opposite evidences on the relation between inserting speed and force. Brett et al. [134] found that the insertion force can be reduced by increasing the needle speed. However, Frick et al. [132] found that the insertion force is unchanged when the moving speed of the needle increases from 1 to $10 \mathrm{~mm} / \mathrm{s}$. In our corresponding studies, the similar contradictory phenomena were also observed. This may be correlated with the sophisticated properties of skin [73].

In conclusion, with the development of micro/nano manufacturing technology and more detailed studies on the corresponding mechanics problems concerning MNs, it is believed that MNs technology will bring us many new exciting breakthroughs in medical, healthcare and biological applications.

\section{Acknowledgements}

This work was supported by National Basic Research Program of China (2015CB057306), National Science Foundation of China (11572080), and the Fundamental Research Funds for the Central Universities (DUT15QY18, DUT15JJG06).

\section{Reference}

[1] M.R. Prausnitz. Microneedles for transdermal drug delivery, Adv. Drug Deliv. Rev. 56 (2004) 581-587.

[2] G.M. Glenn, R.T. Kenney. Mass vaccination: solutions in the skin. In Mass Vaccination: Global Aspects_-Progress and Obstacles. Springer Berlin Heidelberg. (2006) 247-268.

[3] L. Engelke, G. Winter, S. Hook, et al. Recent insights into cutaneous immunization: how to 
vaccinate via the skin, Vaccine 33 (2015) 4663-4674.

[4] M.R. Prausnitz, R. Langer. Transdermal drug delivery, Nat Biotechnol 26 (2008) 1261-1268.

[5] J.W. Wiechers. The barrier function of the skin in relation to percutaneous absorption of drugs, Pharm. Weekblad 11 (1989) 185-198.

[6] Y.B. Choy, M.R. Prausnitz. The rule of five for non-oral routes of drug delivery: ophthalmic, inhalation and transdermal, Pharm. Res. 28 (2011) 943-948.

[7] M.S. Gerstel, V.A. Place. U.S. Patent No. 3,964,482. Washington, DC: U.S. Patent and Trademark Office, 1976.

[8] R.F. Donnelly, T.R.R. Singh, A.D. Woolfson. Microneedle-based drug delivery systems: microfabrication, drug delivery, and safety, Drug Deliv. 17 (2010) 187-207.

[9] S. Henry, D.V. McAllister, M.G. Allen, et al. Microfabricated microneedles: a novel approach to transdermal drug delivery, J. Pharm. Sci. 87 (1998) 922-925.

[10] S. Kaushik, A.H. Hord, D.D. Denson, et al. Lack of pain associated with microfabricated microneedles, Anesth. Analg. 92 (2001) 502-504.

[11] Y.C. Kim, J.H. Park, M.R. Prausnitz. Microneedles for drug and vaccine delivery, Adv. Drug Deliv. Rev. 64 (2012) 1547-1568.

[12] R.F. Donnelly, D. Douroumis. Microneedles for drug and vaccine delivery and patient monitoring, Drug Deliv. Trans. Res. 5 (2015) 311-312.

[13] B.P. Chaudhri, F. Ceyssens, P. De Moor, et al. A high aspect ratio SU-8 fabrication technique for hollow microneedles for transdermal drug delivery and blood extraction. J. Micromech. Microeng. 20 (2010) 064006.

[14] K. Lee, H.C. Lee, D.S. Lee, et al. Drawing lithography: three-dimensional fabrication of an ultrahigh-aspect-ratio microneedle, Adv. Mat. 22 (2010) 483-486.

[15] Z. Xiang, S.C. Yen, N. Xue, et al. Ultra-thin flexible polyimide neural probe embedded in a dissolvable maltose-coated microneedle, J. Micromech. Microeng. 24 (2014) 065015.

[16] J. Jiang, J.S. Moore, H.F. Edelhauser, et al. Intrascleral drug delivery to the eye using hollow microneedles, Pharm. Res. 26 (2009) 395-403.

[17] D. Zhang, D.B. Das, C.D. Rielly. Potential of microneedle-assisted micro-particle delivery by gene guns: a review, Drug Deliv. 21 (2014) 571-587.

[18] K. Yum, N. Wang, M.F. Yu. Nanoneedle: A multifunctional tool for biological studies in living cells, Nanoscale 2 (2010) 363-372.

[19] K. Yum, M.F. Yu, N. Wang, et al. Biofunctionalized nanoneedles for the direct and site-selective delivery of probes into living cells, Biochim. Biophys. Acta. 1810 (2011) 330-338.

[20] L. Ventrelli, L. Marsilio Strambini, G. Barillaro. Microneedles for Transdermal Biosensing: 
Current Picture and Future Direction, Advanced healthcare materials, 4(17) (2015) 2606-2640.

[21] J. Arya, M.R. Prausnitz. Microneedle patches for vaccination in developing countries, J. Contr. Rel. 240 (2015) 135-141.

[22] R.J. Pettis, A.J. Harvey. Microneedle delivery: clinical studies and emerging medical applications, Ther. Deliv. 3 (2012) 357-371.

[23] M.G. Nandagopal, R. Antony, S. Rangabhashiyam, et al. Overview of microneedle system: a third generation transdermal drug delivery approach, Microsyst. Technol. 20 (2014) 1249-1272.

[24] H. Suh, J. Shin, Y.C. Kim. Microneedle patches for vaccine delivery, Clin. Exp. Vaccine Res. 3 (2014) 42-49.

[25] S. Indermun, R. Luttge, Y.E. Choonara, et al. Current advances in the fabrication of microneedles for transdermal delivery, J. Contr. Rel. 185(2014) 130-138.

[26] L. Lin, A.P. Pisano. Silicon-processed microneedles, J. Microelectromech. Syst. 8 (1999) 78-84.

[27] P. Griss, G. Stemme. Side-opened out-of-plane microneedles for microfluidic transdermal liquid transfer, J. Microelectromech. Syst. 12 (2003) 296-301.

[28] J. Wang, J. Lu, S.Y. Ly, et al. Lab-on-a-cable for electrochemical monitoring of phenolic contaminants, Analyt. Chem. 72 (2000) 2659-2663.

[29] D.V. McAllister, P.M. Wang, S.P. Davis, et al. Microfabricated needles for transdermal delivery of macromolecules and nanoparticles: fabrication methods and transport studies, Proc. Natl. Acad. Sci. USA 100 (2003) 13755-13760.

[30] W. Martanto, J.S. Moore, T. Couse, et al. Mechanism of fluid infusion during microneedle insertion and retraction. J. Contr. Rel. 112 (2006) 357-361.

[31] W. Martanto, J.S. Moore, O. Kashlan, et al. Microinfusion using hollow microneedles, Pharm. Res. 23 (2006) 104-113.

[32] A. Ovsianikov, B. Chichkov, P. Mente, et al. Two photon polymerization of polymer-ceramic hybrid materials for transdermal drug delivery, Int. J. Appl. Ceram. Tech. 4 (2007) 22-29.

[33] B. Cai, W. Xia, S. Bredenberg, et al. Bioceramic microneedles with flexible and self-swelling substrate, Eur. J. Pharm. Biopharm. 94 (2015) 404-410.

[34] W. Martanto, S.P. Davis, N.R. Holiday, et al. Transdermal delivery of insulin using microneedles in vivo, Pharm. Res. 21 (2004) 947-952.

[35] K. Kim, J.B. Lee. High aspect ratio tapered hollow metallic microneedle arrays with microfluidic interconnector, Microsyst. Technol. 13 (2007) 231-235.

[36] F.J. Verbaan, S.M. Bal, D.J. Van den Berg, et al. Assembled microneedle arrays enhance the 
transport of compounds varying over a large range of molecular weight across human dermatomed skin, J. Contr. Rel. 117 (2007) 238-245.

[37] M.M. Badran, J. Kuntsche, A. Fahr. Skin penetration enhancement by a microneedle device (Dermaroller®) in vitro: dependency on needle size and applied formulation. Eur. J. Pharm. Sci. 36 (2009) 511-523.

[38] R.F. Donnelly, T.R.R. Singh, M.J. Garland, et al. Hydrogel-forming microneedle arrays for enhanced transdermal drug delivery. Adv. Funct. Mater. 22(2012) 4879-4890.

[39] R.F. Donnelly, K. Moffatt, A.Z. Alkilani, et al. Hydrogel-forming microneedle arrays can be effectively inserted in skin by self-application: A pilot study centred on pharmacist intervention and a patient information leaflet, Pharm. Res. 31 (2014) 1989-1999.

[40] J.H. Park, M.G. Allen, M.R. Prausnitz. Biodegradable polymer microneedles: fabrication, mechanics and transdermal drug delivery, J. Contr. Rel. 104 (2005) 51-66.

[41] F. Sammoura, J. Kang, Y.M. Heo, et al. Polymeric microneedle fabrication using a microinjection molding technique, Microsyst. Technol. 13 (2007) 517-522.

[42] S. Aoyagi, H. Izumi, M. Fukuda. Biodegradable polymer needle with various tip angles and consideration on insertion mechanism of mosquito's proboscis, Sens. Actuators A: Phys. 143 (2008) 20-28.

[43] Y.G. Li, C.S. Yang, J.Q. Liu, et al. Fabrication of a polymer micro needle array by mask-dragging x-ray lithography and alignment x-ray lithography, Chinese Phys. Let. 28 (2011) 038101.

[44] J.W. Lee, M.R. Han, J.H. Park. Polymer microneedles for transdermal drug delivery, J. Drug Targeting 21 (2013) 211-223.

[45] B. Bediz, E. Korkmaz, R. Khilwani, et al. Dissolvable microneedle arrays for intradermal delivery of biologics: fabrication and application, Pharm. Res. 31 (2014) 117-135.

[46] S.P. Sullivan, D.G. Koutsonanos, M. del Pilar Martin, et al. Dissolving polymer microneedle patches for influenza vaccination, Nat. Med. 16 (2010) 915-920.

[47] E.A. Allen, C. O’Mahony, M. Cronin, et al. Dissolvable microneedle fabrication using piezoelectric dispensing technology, Int. J. Pharm. 500 (2016) 1-10.

[48] T. Miyano, Y. Tobinaga, T. Kanno, et al. Sugar micro needles as transdermic drug delivery system, Biomed. Microdevices 7 (2005) 185-188.

[49] R.F. Donnelly, A. Morrissey P.A. McCarron, et al. Microstructured devices for transdermal drug delivery and minimally-invasive patient monitoring, Recent Pat. Drug, Deliv. Formul, 1 (2007) 195-200.

[50] K. Lee, C.Y. Lee, H. Jung. Dissolving microneedles for transdermal drug administration prepared by stepwise controlled drawing of maltose, Biomaterials 32 (2011) 3134-3140. 
[51] A.K. Banga. Microporation applications for enhancing drug delivery. Expert Opin. Drug Deliv. 6 (2009) 343-354.

[52] S. Khumpuang, R. Maeda, S. Sugiyama. Design and fabrication of a coupled microneedle array and insertion guide array for safe penetration through skin. In Micromechatronics and Human Science, 2003. Proceedings of 2003 International Symposium on (pp. 233-237). IEEE.

[53] C.G. Li, C.Y. Lee, K. Lee, et al. An optimized hollow microneedle for minimally invasive blood extraction, Biomed. Microdevices 15 (2013) 17-25.

[54] L.Y. Chu, M.R. Prausnitz. Separable arrowhead microneedles, J. Contr. Rel. 149 (2011) 242-249.

[55] S.J. Paik, S. Byun, J.M. Lim, et al. In-plane single-crystal-silicon microneedles for minimally invasive microfluid systems, Sens. Actuators A: Phys. 114 (2004) 276-284.

[56] G. Yan, K.S. Warner, J. Zhang, et al. Evaluation needle length and density of microneedle arrays in the pretreatment of skin for transdermal drug delivery, Int. J. Pharm. 391 (2010) $7-12$.

[57] M.G. Zuck. U.S. Patent No. 6,050,988. Washington, DC: U.S. Patent and Trademark Office, 2000.

[58] M.J. Cormier, A.S. Nat, A.P. Neukermans, et al. U.S. Patent No. 6,230,051. Washington, DC: U.S. Patent and Trademark Office, 2001.

[59] J.H. Oh, H.H. Park, K.Y. Do, et al. Influence of the delivery systems using a microneedle array on the permeation of a hydrophilic molecule, calcein, Eur. J. Pharm. Biopharm. 69 (2008) 1040-1045.

[60] H.J. Gardeniers, R. Luttge, E.J. Berenschot, et al. Silicon micromachined hollow microneedles for transdermal liquid transport, J. Microelectromech. Syst, 12 (2003) 855-862.

[61] J.A. Matriano, M. Cormier, J. Johnson, et al. Macroflux ${ }^{\circledR}$ microprojection array patch technology: a new and efficient approach for intracutaneous immunization, Pharm. Res. 19 (2002) 63-70.

[62] J. Min, J.H. Park, H.H. Yoon, et al. Ultrasonic welding method to fabricate polymer microstructure encapsulating protein with minimum damage, Macromolecular Res. 16 (2008) 570-573.

[63] J.H. Park, S.O. Choi, R. Kamath, et al. Polymer particle-based micromolding to fabricate novel microstructures, Biomed. Microdevices 9 (2007) 223-234.

[64] H.R. Jeong, H.S. Lee, I.J. Choi, J.H. Park. Considerations in the use of microneedles: pain, convenience, anxiety and safety, J. Drug Targeting, 2016, (just-accepted, available online).

[65] C. Simone, A.M. Okamura. Modeling of needle insertion forces for robot-assisted 
percutaneous therapy. In Robotics and Automation, 2002. Proceedings. ICRA'02. IEEE International Conference on (Vol. 2, pp. 2085-2091).

[66] A.M. Okamura, C. Simone, M.D. O'Leary. Force modeling for needle insertion into soft tissue, IEEE Trans. Biomed. Eng. 51 (2004) 1707-1716.

[67] O.A. Shergold, N.A. Fleck. Mechanisms of deep penetration of soft solids, with application to the injection and wounding of skin, Proc R Soc London A: Math, Phys. Eng. Sci. 460 (2004) 3037-3058.

[68] N. Abolhassani, R. Patel, M. Moallem. Needle insertion into soft tissue: A survey, Med. Eng. \& Phys. 29 (2007) 413-431.

[69] S.P. Davis, B.J. Landis, Z.H. Adams, et al. (2004). Insertion of microneedles into skin: measurement and prediction of insertion force and needle fracture force. $\mathrm{J}$ Biomech 37:1155-1163.

[70] N. Roxhed, T.C. Gasser, P. Griss, et al. Penetration-enhanced ultrasharp microneedles and prediction on skin interaction for efficient transdermal drug delivery, J. Microelectromech. Syst. 16 (2007) 1429-1440.

[71] P. Khanna, K. Luongo, J.A. Strom, et al. Sharpening of hollow silicon microneedles to reduce skin penetration force, J. Micromech. Microeng. 20 (2010) 045011.

[72] R.J. Roark, W.C. Young. Formulas for stress and strain. McGraw-Hill, 1975.

[73] G.L. Wilkes, I.A. Brown, R.H. Wildnauer. The biomechanical properties of skin. CRC critical reviews, Bioengineering 1 (1973) 453.

[74] N. Wilke, A. Mulcahy, S.R. Ye, et al. Process optimization and characterization of silicon microneedles fabricated by wet etch technology, Microelectronics J. 36 (2005) 650-656.

[75] K. Kim, D.S. Park, H.M. Lu, et al. A tapered hollow metallic microneedle array using backside exposure of SU-8, J. Micromech. Microeng. 14 (2004) 597-603.

[76] S. Chandrasekaran, A.B. Frazier. Characterization of surface micromachined hollow metallic microneedles. In Micro Electro Mechanical Systems, MEMS-03 Kyoto. IEEE The Sixteenth Annual International Conference, (2003) 363-366.

[77] P.M. Wang, M. Cornwell, J. Hill, et al. Precise microinjection into skin using hollow microneedles, J. Invest. Dermatol. 126 (2006) 1080-1087.

[78] T. Kawashima, T. Sakai, N. Kato, et al. Mechanical characterization and insertion performance of hollow microneedle array for cell surgery, J Micro/Nanolithography, MEMS, and MOEMS 8 (2009) 033014.

[79] F.J. Verbaan, S.M. Bal, D.J. Van den Berg, et al. Improved piercing of microneedle arrays in dermatomed human skin by an impact insertion method, J. Contr. Rel. 128 (2008) 80-88.

[80] X.Q. Kong, C.W. WU. Measurement and prediction of insertion force for the mosquito 
fascicle penetrating into human skin, J. Bionic Eng. 6 (2009) 143-152.

[81] W.Z. Li, M.R. Huo, J.P. Zhou, et al. Super-short solid silicon microneedles for transdermal drug delivery applications, Int. J. Pharm. 389 (2010) 122-129.

[82] G. Widera, J. Johnson, L. Kim, et al. Effect of delivery parameters on immunization to ovalbumin following intracutaneous administration by a coated microneedle array patch system, Vaccine 24 (2006) 1653-1664.

[83] G. Li, A. Badkar, S. Nema, et al. In vitro transdermal delivery of therapeutic antibodies using maltose microneedles, Int. J. Pharm. 368 (2009) 109-115.

[84] Y.A. Gomaa, D.I. Morrow, M.J. Garland, et al. Effects of microneedle length, density, insertion time and multiple applications on human skin barrier function: assessments by transepidermal water loss, Toxicology in Vitro 24 (2010) 1971-1978.

[85] S.M. Bal, A.C. Kruithof, R. Zwier, et al. Influence of microneedle shape on the transport of a fluorescent dye into human skin in vivo, J. Contr. Rel. 147 (2010) 218-224.

[86] T. Watanabe, K. Hagino, T. Sato. Evaluation of the effect of polymeric microneedle arrays of varying geometries in combination with a high-velocity applicator on skin permeability and irritation, Biomed. Microdevices 16 (2014) 591-597.

[87] D.D. Zhu, Q.L. Wang, X.B. Liu, et al. Rapidly Separating Microneedles for Transdermal Drug Delivery, Acta Biomaterialia 41 (2016) 312-319.

[88] J.A. Schetz, A.E. Fuhs. Fundamentals of fluid mechanics, John Wiley \& Sons, 1999.

[89] R.L. Hood, M.A. Kosoglu, M. Parker, et al. Effects of microneedle design parameters on hydraulic resistance, J. Med. Devices 5 (2011) 031012.

[90] J. Gupta, S.S. Park, B. Bondy B, et al. Infusion pressure and pain during microneedle injection into skin of human subjects, Biomaterials 32 (2011) 6823-6831.

[91] H. Le-Thanh, N. Tran-Minh, H. Le-The, et al. A novel design of hollow microneedle for blood sample collection. In Nano/Micro Engineered and Molecular Systems (NEMS), 2014 9th IEEE International Conference on (pp. 430-435).

[92] P. Schipper, K. van der Maaden, S. Romeijn, et al. Determination of depth-dependent intradermal immunogenicity of adjuvanted inactivated polio vaccine delivered by microinjections via hollow microneedles, Pharm. Res, 33 (2016) 2269-2279.

[93] H.S. Gill, D.D. Denson, B.A. Burris, et al. Effect of microneedle design on pain in human subjects, Clin. J. Pain 24 (2008) 585.

[94] S.M. Bal, J. Caussin, S. Pavel, et al. In vivo assessment of safety of microneedle arrays in human skin, Eur. J. Pharm. Sci. 35 (2008) 193-202.

[95] M.I. Haq, E. Smith, D.N. John et al. Clinical administration of microneedles: skin puncture, pain and sensation, Biomed. Microdevices 11 (2009) 35-47. 
[96] B. Sezgin, B. Ozel, H. Bulam, et al. The effect of microneedle thickness on pain during minimally invasive facial procedures: a clinical study, Aesthetic Surgery J. 34 (2014) 757-765.

[97] J. Gupta, E.I. Felner, M.R. Prausnitz. Minimally invasive insulin delivery in subjects with type 1 diabetes using hollow microneedles, Diab. Technol. Ther. 11 (2009) 329-337.

[98] J. Gupta, D.D. Denson, E.I. Felner, et al. Rapid local anesthesia in human subjects using minimally invasive microneedles, Clin. J. Pain 28 (2012) 129-135.

[99] J. Gupta, E.I. Felner, M.R. Prausnitz. Rapid pharmacokinetics of intradermal insulin administered using microneedles in type 1 diabetes subjects, Diab. Technol. Ther. 13 (2011) 451-456.

[100] P. Van Damme, F. Oosterhuis-Kafeja, M. Van der Wielen, et al. Safety and efficacy of a novel microneedle device for dose sparing intradermal influenza vaccination in healthy adults, Vaccine 27 (2009) 454-459.

[101] N. Becker, D. Petrić, C. Boase, et al. Mosquitoes and their control (Vol. 2). New York: Springer, 2003.

[102] I. Wahid, T. Sunahara, M. Mogi. Maxillae and mandibles of male mosquitoes and female autogenous mosquitoes (Diptera: Culicidae), J. Med. Entomol. 40 (2003) 150-158.

[103] G.G. Robinson. The mouthparts and their function in the female mosquito, Anopheles maculipennis, Parasitology 31 (1939) 212-242.

[104] R.E. Snodgrass. The anatomical life of the mosquito, Smithsonian Miscellaneous Collections 139 (1959) 1-87.

[105] H.W. Krenn, H. Aspöck. Form, function and evolution of the mouthparts of blood-feeding Arthropoda, Arthropod Struct. Dev. 41 (2012) 101-118.

[106] X.Q. Kong. Study on Mechanics Behaviors of Mosquito's Floating and Acupuncturing, Thesis (PhD). Dalian University of Technology, China, 2010.

[107] X.Q. Kong, C.W. WU. Mosquito proboscis: An elegant biomicroelectromechanical system, Phys. Rev. E 82 (2010) 011910.

[108] R.M. Gordon, W.H.R. Lumsden. A study of the behaviour of the mouth-parts of mosquitoes when taking up blood from living tissue; together with some observations on the ingestion of microfilariae, Ann. Trop. Med. Parasit. 33 (1939) 259-278.

[109] H. Izumi, M. Suzuki, S. Aoyagi, et al. Realistic imitation of mosquito's proboscis: electrochemically etched sharp and jagged needles and their cooperative inserting motion, Sens. Actuators A: Phys. 165 (2011) 115-123.

[110] M.K. Ramasubramanian, O.M. Barham, V. Swaminathan. Mechanics of a mosquito bite with applications to microneedle design, Bioinspiration \& biomimetics 3 (2008) 046001. 
[111] K. Oka, S. Aoyagi, Y. Arai, et al. Fabrication of a micro needle for a trace blood test, Sens. Actuators A: Phys. 97 (2002) 478-485.

[112] J. Margalit, R. Galun, M.J. Rice. Mouthpart sensilla of the tsetse fly and their function: I: Feeding patterns, Ann. Trop. Med. Parasit. 66 (1972) 525-536.

[113] M.J. Rice, R. Galun, J. Margalit. Mouthpart sensilla of the tsetse fly and their function: II: Labial sensilla, Ann. Trop. Med. Parasit. 67 (1973) 101-107.

[114] R.A. Leopold, T.P. Freeman, J.S. Buckner, et al. Mouthpart morphology and stylet penetration of host plants by the glassy-winged sharpshooter, Homalodisca coagulata,(Homoptera: Cicadellidae), Arthropod Struct. Dev. 32 (2003) 189-199.

[115] Z.L. Zhao, H.P. Zhao, G.J. Ma, et al. Structures, properties, and functions of the stings of honey bees and paper wasps: a comparative study, Biology Open 4 (2015) 921-928.

[116] G.J. Ma, L.T. Shi, C.W. WU. Biomechanical property of a natural microneedle: the caterpillar spine, J. Med. Dev. 5 (2011) 034502.

[117] J.M. Gere, S.P. Timoshenko. Mechanics of materials Brooks. Cole, Pacific Grove, CA, (2001) 815-39.

[118] W.K. Cho, J.A. Ankrum, D. Guo, et al. Microstructured barbs on the North American porcupine quill enable easy tissue penetration and difficult removal, Proc, Natl, Acad, Sci, USA 109 (2012) 21289-21294.

[119] M. Yang, J.D. Zahn. Microneedle insertion force reduction using vibratory actuation, Biomed. Microdevices 6 (2004) 177-182.

[120] X. Qi. Study of Bionic Needle with both Drag Reduction and Antibacteria Based on Insect Mouthpart, Thesis (PhD). Jilin University, China, 2014.

[121] X. Qi, Y.C. Qi, Y. Li, et al. The insect fascicle morphology research and bionic needle pierced mechanical mechanism analysis, Adv. Nat. Sci. 3 (2010) 251-257.

[122] J.C. Wang. Coupling Bionic Research on Painless Injector Needles Based on Insect Piercing-sucking Mouthpart, Thesis (PhD). Jilin University, China, (2008).

[123] J. Jiang, H.S. Gill, D. Ghate, et al. Coated microneedles for drug delivery to the eye, Invest. Ophthalmol. Vis. Sci. 48 (2007) 4038-4043.

[124] S.R. Patel, D.E. Berezovsky, B.E. McCarey, et al. Targeted administration into the suprachoroidal space using a microneedle for drug delivery to the posterior segment of the EyeMicroneedle use for targeted drug delivery, Invest Ophthalmol Visual Science 53 (2012) 4433-4441.

[125] S.R. Patel, A.S. Lin, H.F. Edelhauser, et al. Suprachoroidal drug delivery to the back of the eye using hollow microneedles, Pharm. Res. 28 (2011) 166-176.

[126] T.R. Thrimawithana, S. Young, C.R. Bunt, et al. Drug delivery to the posterior segment 
of the eye, Drug Discovery Today 16 (2011) 270-277.

[127] M.R. Ahmad, M. Nakajima, S. Kojima, et al. Buckling nanoneedle for characterizing single cells mechanics inside environmental SEM, IEEE Trans. Biomed. Eng. 10 (2011) 226-236.

[128] S.W. Han, C. Nakamura, Y. Imai, et al. Monitoring of hormonal drug effect in a single breast cancer cell using an estrogen responsive GFP reporter vector delivered by a nanoneedle, Biosensors and Bioelectronics 24 (2009) 1219-1222.

[129] Z. Liu, S. Tabakman, K. Welsher, et al. Carbon nanotubes in biology and medicine: in vitro and in vivo detection, imaging and drug delivery, Nano Res 2 (2009) 85-120.

[130] C. Chiappini, E. De Rosa, J.O. Martinez, et al. Biodegradable silicon nanoneedles delivering nucleic acids intracellularly induce localized in vivo neovascularization, Nat. Mat. 14 (2015) 532-539.

[131] S. Sharma, A. Saeed, C. Johnson, et al. Rapid, low cost prototyping of transdermal devices for personal healthcare monitoring, Sensing and Bio-Sensing Res. 2016, (just-accepted , available on-line http://www.sciencedirect.com/science/article/pii/S221418 $\underline{0416301490})$.

[132] T.B. Frick, D.D. Marucci, J.A. Cartmill, et al. Resistance forces acting on suture needles, J. Biomech. 34 (2001) 1335-1340.

[133] F.H. Figge, D.J. Barnett. Anatomic evaluation of a jet injection instrument designed to minimize pain and inconvenience of parenteral therapy, J. Am. Acad. Nurse Pract. 3 (1948) 197-206.

[134] P.N. Brett, T.J. Parker, A.J. Harrison, et al. Simulation of resistance forces acting on surgical needles, Proc. Instn. Mech. Engrs. Part H: J. Eng. Med. 211 (1997) 335-347. 


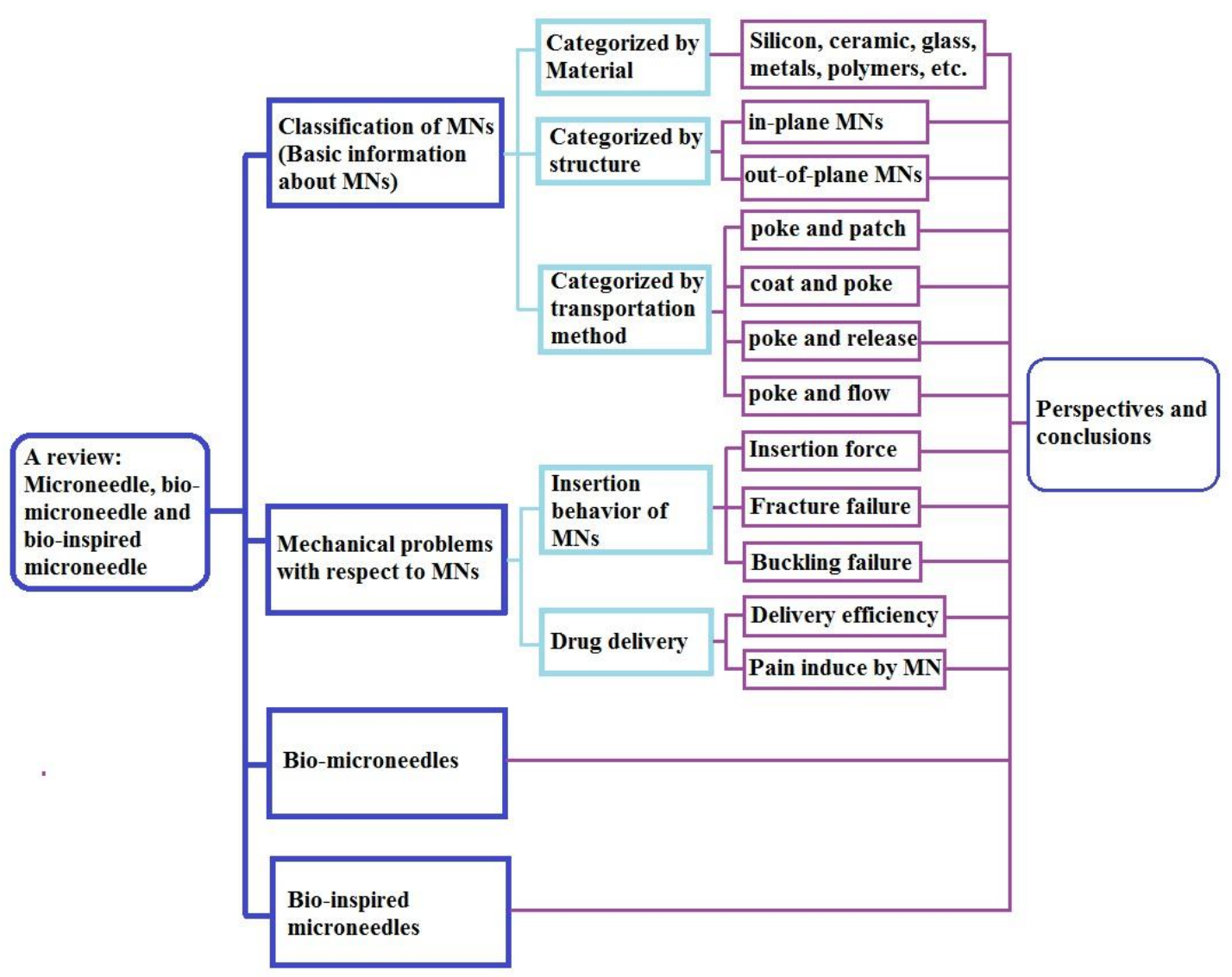

